\title{
Evaluation of methods for calculating volume fraction in Eulerian-Lagrangian multiphase flow simulations
}

\author{
Angela Diggs ${ }^{a, b} \& S$. Balachandar ${ }^{\text {a } 1}$ \\ aDepartment of Mechanical \& Aerospace Engineering, University of Florida, Gainesville, FL, \\ 32611; ' Air Force Research Laboratory, Munitions Directorate, Eglin AFB, FL, 32542
}

\begin{abstract}
The present work addresses numerical methods required to compute particle volume fraction or number density. Local volume fraction of the $l^{\text {th }}$ particle, $\alpha_{l}$, is the quantity of foremost importance in calculating the gas-mediated particle-particle interaction effect in multiphase flows. A general multiphase flow with a distribution of Lagrangian particles inside a fluid flow discretized on an Eulerian grid is considered. Particle volume fraction is needed both as a Lagrangian quantity associated with each particle and also as an Eulerian quantity associated with the grid cell for Eulerian-Lagrangian simulations. In Grid-Based (GB) methods the particle volume fraction is first obtained within each grid cell as an Eulerian quantity and then the local particle volume fraction associated with any Lagrangian particle can be obtained from interpolation. The second class of methods presented are Particle-Based (PB) methods, where particle volume fraction will first be obtained at each particle as a Lagrangian quantity, which then can be projected onto the Eulerian grid. Traditionally, the GB methods are used in multiphase flow, but sub-grid resolution can be obtained through use of the PB methods. By evaluating the total error, and its discretization, bias and statistical error components, the performance of the different PB methods are compared against several common GB methods of calculating volume fraction. The standard von Neumann error analysis technique has been adapted for evaluation of rate of convergence of the different methods. The discussion and error analysis presented focus on the volume fraction calculation, but the methods can be extended to obtain field representations of other Lagrangian quantities, such as particle velocity and temperature.
\end{abstract}

Distribution A. Approved for public release, distribution is unlimited.

\section{Introduction}

The Eulerian-Lagrangian point-particle approach has become an important methodology in the simulation of canonical and complex multiphase flows [1, 2, 3]. In this approach the continuous carrier phase is solved in the Eulerian frame of reference, typically using a fixed Eulerian grid, while the time evolution of the dispersed phase (can be particles, droplets or bubbles) is considered in the Lagrangian frame by tracking every individual particle's position, velocity and other properties ${ }^{2}$.

\footnotetext{
${ }^{1}$ Corresponding author. Tel: +1 3523928909 ; fax +1 3523927303

Email address: bala1s@ufl.edu (S. Balachandar)

${ }^{2}$ Henceforth the term "particles" will apply equally well to droplets and bubbles.
} 
There are four key components to a general Eulerian-Lagrangian point-particle simulation: 1) Fluid-fluid interaction represented by the continuous phase Navier-Stokes equation, whose solution on an Eulerian grid is the subject matter of standard CFD. 2) Fluid-to-particle forward coupling which accounts for the effect of the continuous phase on the dispersed phase. 3) Particle-to-fluid coupling which accounts for the back effect of particles on the fluid. 4) Particle-particle coupling which accounts for the interaction between the particles, either through direct collisions or as mediated by the continuous phase $[4,5,6,7$, 8]. In one-way coupled simulations only the first two interactions are modelled and in two-way coupled simulations particle-to-fluid back coupling is also included. When particle-particle interactions are important, the multiphase flow problem is considered to be four-way coupled $[9,10]$.

Fluid-to-particle coupling requires interpolation of the Eulerian continuous phase properties to the location of the Lagrangian particles, which in general will not coincide with the Eulerian grid points. A number of interpolation schemes, such as linear interpolation, fourth and sixth order Lagrange interpolation, and Hermite interpolation, have been proposed and their accuracies analyzed [11, 12].

Particle-to-fluid coupling involves projection of the Lagrangian quantities, such as particle drag force and heat transfer, back to the continuous phase momentum and energy equations as source terms. This projection is from the position of the Lagrangian particles to the Eulerian grid points. Due to conservation of momentum and energy, the projection operation must satisfy partition of unity - in other words, the total feedback of momentum and energy from each particle to the surrounding Eulerian grid must be equal and opposite to those of the particle. The following three projection schemes have been widely considered in the past: 1) particle-in-cell (PIC) method pioneered by Evans et al. [13] and Harlow [14], 2) projection on neighboring node method [1, 2] and 3) projection onto identical stencil method advanced by Sundaram and Collins [15]. The accuracy and relative performance of the different projection methods have been considered by Boivin et al. [16] and Narayanan et al. [17].

Of particular significance is the work of Garg et al. [18] who presented a rigorous analysis of the different projection operations in conjunction with different interpolation operations and separated the error into deterministic (discretization and bias errors) and stochastic (statistical error) components. They demonstrated it is important for numerical convergence to maintain the number of Lagrangian points per grid cell while performing a grid refinement study.

The focus of the present work is to extend the above investigations and consider an aspect of fluidmediated particle-particle interaction (i.e., the effect of neighboring particles on quantities such as particle drag force). Different methods that are used in the computation of particle volume fraction will be considered and their accuracy evaluated. Local particle volume fraction around the $l^{\text {th }}$ particle, $\alpha_{l}$, and the volume fraction at the $i^{\text {th }}$ grid cell are the quantities of foremost importance. These information are often required in the modeling fluid-mediated particle-particle interaction and back coupling of momentum to the carrier fluid. Local volume fraction can be defined in different ways. Particle volume fraction is traditionally defined as the fractional volume occupied by the particles inside a reference volume, whose size is chosen to be much smaller than the scale of volume fraction variation. In this definition local volume fraction around any particle depends on the distribution of a cloud of particles around it within the reference volume. On the other hand, given the location of all the particles within the flow domain, one can consider Voronoi tessellation of the entire domain and define the volume of the Voronoi element around each particle to be the volume of space associated with that particle [19]. Then the local particle volume fraction of a particle can be defined as the ratio of the particle volume to 
the volume of space associated with it. In this later definition, local particle volume fraction depends only on the location of the nearest neighbors. Irrespective of the precise definition, it is clear that particle volume fraction is fundamental information that depends on inter-particle distances.

Here a general multiphase flow with a distribution of Lagrangian particles inside a fluid flow discretized on an Eulerian grid is considered. Numerical methods for the evaluation of particle volume fraction are addressed. In Eulerian-Lagrangian simulations particle volume fraction is needed both as a Lagrangian quantity associated with each particle and also as an Eulerian quantity associated with the grid cell. For instance, correlations have been proposed to account for the effect of volume fraction on the drag force on a particle. Thus, in the evaluation of drag force, local particle volume fraction is needed as a Lagrangian quantity. On the other hand, in the continuous phase governing equations in order to account for the spatial variation of the fluid volume fraction, it is also necessary to represent particle volume fraction as an Eulerian quantity (note by definition the sum of the local particle and fluid volume fraction is unity). Clearly it is sufficient to first obtain particle volume fraction either in the Lagrangian frame for each particle or in the Eulerian frame as a field variable. Once one of these two representations is known interpolation or projection techniques can be used to obtain the other representation.

Two classes of methods for the evaluation of particle volume fraction are addressed. The first will be termed Grid-Based (GB) methods, where particle volume fraction will first be obtained within each grid cell as an Eulerian quantity, from which local particle volume fraction associated with any Lagrangian particle can be obtained from interpolation. The second class of methods will be termed Particle-Based (PB) methods, where particle volume fraction will first be obtained at each particle as a Lagrangian quantity, which can then be projected onto the Eulerian grid.

The traditional approach to particle volume fraction evaluation has been using GB methods. For instance, the lowest order GB method to calculate particle volume fraction is to count all particles within a grid cell and add their volume contributions to obtain the local particle volume fraction within that cell. At the next level, each particle contributes part of its volume to the particle volume fraction of the grid cells surrounding it, according to its location with respect to the surrounding grid cell centers. By construction, the GB methods can represent particle volume fraction variation only on the grid scaleany volume fraction variation on scales smaller than the grid is erased in the grid-scale averaging process.

As pointed out by Garg et al. $[18,20]$ in regions where there are particles it is necessary to have a sufficiently large number of particles per cell to reduce statistical and deterministic errors in particle-tofluid back coupling. The bias error decreases as inverse of the number of particles per cell and the statistical error decreases as the square root of the inverse of the number of particles per cell. It can be argued that having more than one particle per cell offers sub-grid resolution, which can be exploited in evaluating Lagrangian quantities at a finer scale than the grid cell (here sub-grid indicates resolution at a scale finer than the Eulerian grid used for the carrier fluid). The degree of sub-grid resolution will be dictated by the number and distribution of particles within and in the neighborhood of a cell.

Here PB methods are presented for the evaluation of particle volume fraction as a Lagrangian quantity. These methods rely only upon the location of the neighboring particles and are completely independent of the Eulerian grid. As a result, they offer potential for sub-grid resolution, as the number of particles per cell increases. By evaluating the total error, and its discretization, bias and statistical error 
components, the performance of the different PB methods will be compared against the corresponding $\mathrm{GB}$ methods for evaluating particle volume fraction. The standard von Neumann error analysis technique will be adapted for rigorous evaluation of rate of convergence of the different methods. Thus, the number of grid cells per wave and the number of Lagrangian particles per cell are the key variables in the error analysis.

An important point to be stressed is that the present analysis applies to only low volume fraction, since it concerns only single-point statistics. The random distribution of particles represented as points in space is described by the theory of stochastic point processes [21] (for application to multiphase flows see $[22,23,24])$. The Poisson point process used in generating the random particle locations in the present study implicitly assumes complete independence and thus applies only to low volume fraction. With increasing volume fraction the analysis must employ Matern point process that accounts for volume exclusion and other nearest neighbor effects.

The discussion to be presented below will focus only on particle volume fraction evaluation. But the PB methods to be presented and discussed can be extended to obtain field representations of other Lagrangian quantities such as particle velocity, temperature, etc. The resulting field representation will be at a scale dictated by the number and distribution of the Lagrangian particles, rather than the Eulerian grid, thus permitting resolution at scales smaller than the Eulerian grid. For inertial particles, such sub-grid resolution is highly desired since it is now well established that particle volume fraction (or concentration or number density) can vary on length scales much finer than the Kolmogorov scale of underlying turbulence $[25,26]$. Thus, with PB methods one can address particle-particle interaction processes that occur at fine scale, while the collective back coupling of particles on the fluid can be represented through the conventional projection operation to the Eulerian grid. This coarse graining to the Kolmogorov scale is sufficient for representing momentum and energy feedback of particles to the flow, since smaller flow scales will be rapidly dissipated.

\section{Methodology}

\subsection{Volume fraction evaluation for Lagrangian particles}

In order to evaluate and compare the accuracy of the GB and PB methods, the local particle volume fraction calculated using a finite number of Lagrangian particles within a computational domain is considered. We prescribe a sinusoidal spatial variation in volume fraction, $\alpha(x)$, and consider this to be given (or known) from a cloud of very large number of very small particles. We now randomly distribute a small finite number of Lagrangian particles according to this spatially varying volume fraction and calculate the volume fraction variation back from these finite number of Lagrangian particles using GB and PB methods. This will allow us to directly assess the accuracy of each GB and PB method proposed.

Consider a one-dimensional periodic domain of length $2 \pi$ discretized into $N$ equal Eulerian grid cells of thickness $\Delta X=2 \pi / N$. Grid resolution may be varied by changing the number of grid cells, $N$. The domain will be considered periodic and of unit width along the $y$ and $z$ directions. A total number of $N M$ particles within the domain are considered, which yields the average number of particles within each grid cell, $M$. For any combination of $N$ and $M$, the exact volume fraction variation is defined as

$$
\alpha_{\text {exact }}(x)=\alpha_{\text {avg }}\left(1+\alpha_{1} \cos [x]\right),
$$




$$
\alpha_{\text {avg }}=N M V / 2 \pi
$$

where $V$ is the volume of a particle, and $\alpha_{1}$ is the relative amplitude of the sinusoidal variation (since volume fraction is a non-negative quantity, $0 \leq \alpha_{1} \leq 1$ ). For each combination of $N$ and $M$, the particles are distributed randomly within the domain according to the probability $P(x)$, using the usual definition that $P(x) d x$ is the probability of finding the particle in the region $[x-d x / 2, x+d x / 2]$. With the probability given by the normalized volume fraction variation, the cumulative density function $C(x)$ is defined and used to randomly place the $N M$ particles within the domain

$$
P(x)=\frac{1}{2 \pi}\left(1+\alpha_{1} \cos [x]\right) \text { and } C(x)=\int_{0}^{x} P(\varepsilon) d \varepsilon=\frac{1}{2 \pi}\left(x+\alpha_{1} \sin [x]\right) .
$$

Each random distribution of $N M$ particles will be termed a realization and for each realization numerical approximation to the local volume fraction will be obtained using the GB or PB methods. For the GB methods, the local particle volume fraction at each grid cell is first calculated, then interpolated to each Lagrangian particle; the same order of approximation for the projection and interpolation operations is used. For the PB methods, the local particle volume fraction at each Lagrangian particle is first calculated, then projected to each grid cell. The error of each method is assessed by comparing the exact volume fraction with the calculated volume fraction at each particle location (note the error could also be calculated by comparing the actual and calculated volume fractions within each Eulerian cell as well). The GB and PB methods considered are outlined below.

\subsection{Grid-based methods for particle volume fraction evaluation}

In general, particle volume fraction calculated from GB methods involves summing or weighting the contribution of the Lagrangian particles within a given cell (zeroth order), between cell centers (first order), and so on. For simplicity finite volume based methods are considered, but the discussion can be easily extended to finite difference and other spatial discretization methods. The volume fraction value for the Eulerian cell is stored at the cell center, and when local volume fraction is needed at a Lagrangian particle, the Eulerian volume fraction value is interpolated to the particle's location, typically using the same order method as used for the volume fraction calculation.

\subsubsection{Zeroth order method}

In the zeroth order method, the particles contribute directly to the Eulerian grid cell where they reside, where the faces for cell $i$ are $X_{i-1 / 2}$ and $X_{i+1 / 2}$. If a particle at location $x_{j}$ resides with in cell $i$, the weight of that particle on cell $i$ is $1 ; 0$ otherwise. The weights of all particles over all cells is summed to find the total number of particles per cell, $m_{0}$. The particle volume fraction of each Eulerian grid cell is calculated as

$$
\begin{aligned}
& W_{0, i}\left(x_{j}\right)= \begin{cases}1 & \text { if } X_{i-1 / 2} \leq x_{j} \leq X_{i+1 / 2} \\
0 & \text { otherwise } .\end{cases} \\
& m_{0, i}=\sum_{k=1}^{N M} W_{0, i}\left(x_{k}\right), \\
& \alpha_{G 0, i}=\alpha_{a v g} \frac{m_{0, i}}{M} .
\end{aligned}
$$

Interpolation to the Lagrangian particles is quite simple for the zeroth order method; the particles within a given cell simply take the particle volume fraction value of the particular cell. 


$$
\alpha_{P 0, j}=\alpha_{G 0, i} \quad \text { if } \quad X_{i-1 / 2}<x_{j}<X_{i+1 / 2} .
$$

\subsubsection{First order method}

In the first order method, the particles contribute to the two cells whose centers enclose the particle. The particle's non-dimensional location, $\xi_{j}$, is calculated as a function of its distance from the two cell centers, $X_{i}$ and $X_{i+1}$, where the mean particle spacing, $\Delta x=2 \pi /(N M)$, is used to non-dimensionalize. The first order weight function, $W_{1, i}(\xi)$, distributes the particle's influence between the two cells, where the total influence of a single particle must sum to unity. The total number of particles within each cell is the summation of the weight function for that cell over all particles. Similar to the zeroth order method, the local particle volume fraction of each cell is calculated as

$$
\begin{aligned}
& \xi_{j i}=\left(x_{j}-X_{i}\right) / \Delta x, \\
& W_{1, i}\left(\xi_{j i}\right)=\left\{\begin{array}{cc}
1+\frac{\xi_{j i}}{M} & \text { if }-M \leq \xi_{j i} \leq 0 \\
1-\frac{\xi_{j i}}{M} & \text { if } 0 \leq \xi_{j i} \leq M \\
0 & \text { otherwise }
\end{array}\right. \\
& m_{1, i}=\sum_{k=1}^{N M} W_{1, i}\left(\xi_{k i}\right), \\
& \alpha_{G 1, i}=\alpha_{\text {avg }} \frac{m_{1, i}}{M} .
\end{aligned}
$$

The first order interpolation from Eulerian cell to Lagrangian particles requires the use of a linear weight function dependent on the particle's location between the nearest cell centers.

$$
\alpha_{P 1, j}=\alpha_{G 1, i} w+\alpha_{G 1, i+1}(1-w) \text { if } X_{i}<x_{j}<X_{i+1},
$$

where the weight is defined as $w=X_{i+1}-x_{j} / \Delta X$.

\subsubsection{Second order method}

In the second order method, the discrete delta function $[27,28]$ is employed, such that each particle contributes to local volume fraction of three cells: the cell where it currently resides, and the two adjoining cells. The influence (or weight) the particle has on each cell is determined by the generator function, $\psi(r)$, where $r=\xi_{j i} / M$. Though spread across three cells, the weight of each particle sums to 1 , satisfying the partition of unity requirement. The total number of particles in each cell, $m_{2, i}$, is found as the summation of the influence of all the particles. The particle volume fraction of each cell is again calculated as

$$
\begin{aligned}
& \psi(r)=\left\{\begin{array}{lr}
\frac{1}{6}\left(5-3|r|-\sqrt{-3\left(1-|r|^{2}+1\right)}\right) & \text { if } \\
\frac{1}{3}\left(1+\sqrt{-3 r^{2}+1}\right) & \text { if }|r| \leq 0.5 \\
0 & \text { otherwise }
\end{array}\right. \\
& W_{2, i}\left(\xi_{j i}\right)=\psi\left(\frac{\xi_{j i}}{M}\right), \\
& m_{2, i}=\sum_{k=1}^{N M} W_{2, i}\left(\xi_{k i}\right), \\
& \alpha_{G 2, i}=\alpha_{\text {avg }} \frac{m_{2, i}}{M} .
\end{aligned}
$$


Although there are other second order projection operators that allow each particle to contribute to the three nearest grid cells, the above discrete delta function has the advantage that it precisely satisfies partition of unity and zero net moment. It is widely used in the implementation of immersed boundary methodology $[27,28,29]$ and has been adopted here. The second order interpolation from Eulerian cell to Lagrangian particles also uses the discrete delta function, where the particle volume fraction is the summation of the Eulerian volume fraction and generator function over all grid cells.

$$
\alpha_{P 2, j}=\sum_{i=1}^{N} \alpha_{G 2, i} \psi\left(\frac{\xi_{j i}}{M}\right) .
$$

\subsection{Particle-based methods for particle volume fraction evaluation}

In general, PB methods of calculating particle volume fraction are independent of the underlying Eulerian grid. The local volume fraction of each particle depends only on the number and location of its neighboring particles. Thus, the PB methods to be considered here are similar those used in mesh-free (or mesh-less) methods [30].

\subsubsection{Top-hat method}

The top-hat PB method of calculating local particle volume fraction considers all the particles within a region around a reference particle, $\left[x_{j}-\delta / 2, x_{j}+\delta / 2\right]$, where $\delta$ is the width of the top-hat filter. All particles within the region are assigned a uniform influence (weight) and the local particle volume fraction is calculated as

$$
\begin{aligned}
& w_{T H, j i}=\left\{\begin{array}{ll}
1 & \text { if } x_{j}-\frac{\delta}{2}<x_{i}<x_{j}+\frac{\delta}{2} \\
0 & \text { otherwise }
\end{array},\right. \\
& m_{T H, j}=\sum_{i=1}^{N M} w_{T H, j i}, \\
& \alpha_{T H, j}=\alpha_{\text {avg }} \frac{m_{T H, j}}{M} .
\end{aligned}
$$

The top-hat method is so named because it gives equal weight to all particles within $\delta$ of the reference particle. The top-hat method can be implemented without reference to the underlying Eulerian grid by specifying $\delta$ to be any desired value independent of the Eulerian grid width. However, in the present context of Eulerian-Lagrangian multiphase flow implementation, which will involve an underlying Eulerian grid for the simulation of the continuous phase, the width $\delta$ may be chosen to be the same as the grid cell width.

\subsubsection{Gaussian method}

For the Gaussian PB method, the equation for the local particle volume fraction is calculated using a Gaussian weighting of the neighboring particles (as opposed to the equal weight assigned in the top-hat method),

$$
\alpha_{G S, j}=\sum_{i=1}^{N M} \frac{1}{\delta \sqrt{\pi}} \exp \left[-\left(\frac{X_{j}-X_{i}}{\delta}\right)^{2}\right],
$$

where $N M$ is the total number of particles, $\delta$ defines the width of the Gaussian filter, $X_{j}-X_{i}$ is the spacing between particle $j$ (reference) and all other $i$ particles. Small values of $\delta$ yield a more narrow Gaussian, where only the location of a few neighboring particles will contribute to local volume fraction evaluation. On the other hand, large values of $\delta$ result in a broad Gaussian and result in a smoothening of the local particle volume fraction variation. 


\subsection{Definition of errors}

In each realization, for a given random distribution of $N M$ particles, the above defined GB and PB methods are used to numerically evaluate the local particle volume fraction. The resulting numerical approximation of the local volume fraction at particle $j\left(\alpha_{n u m, j}\right)$ can be directly compared to the exact local volume fraction $\left(\alpha_{\text {exact }, j}=\alpha_{\text {exact }}\left(x_{j}\right)\right)$ to obtain an error estimate. The mean squared error for a single $(p)$ realization may be calculated as

$$
\bar{e}_{p}=\frac{1}{N M} \sum_{j=1}^{N M}\left[\frac{\left(\alpha_{n u m, j}-\alpha_{\text {exact }, j}\right)}{\alpha_{\text {avg }}}\right]^{2},
$$

from which the rms error can be obtained by taking the square root. Each particle has an error in the computed local volume fraction and in the above expression the overbar in $\bar{e}_{p}$ denotes an average over all the particles. The average thus provides a measure of error for the realization. The realization is repeated many times to construct an ensemble of $P$ realizations. For the ensemble of realizations, the average mean squared error is calculated as

$$
\overline{\bar{e}}=\frac{1}{P} \sum_{p=1}^{P} \bar{e}_{p}
$$

where the double overbar indicates averages over both an ensemble of realizations and over all $N M$ particles within each realization. Following Xu and Pope [31] and Garg et al [18] the overall error is separated into deterministic and stochastic components. For each GB and PB method the error depends on the value of both $N$ and $M$. The limit $M \rightarrow \infty$ will be termed the dense particle limit, and in this limit the statistical sampling error goes to zero, since the averaging is over a dense distribution of a very large number of Lagrangian particles. In this limit the only error is due to Eulerian discretization of the domain into a finite number of grid cells, and thus the discretization error is defined as

$$
\text { Discretization Error: } \quad \overline{\bar{e}}_{D}(N)=[\overline{\bar{e}}]_{M \rightarrow \infty} \text {. }
$$

For GB methods the discretization error depends on the grid resolution $N$ and the order of accuracy of the interpolation and projection schemes. For PB methods the discretization error depends on the tophat or Gaussian averaging operator $\delta$ (filter width). The size of the ensemble is assumed to be sufficiently large (i.e., $P \rightarrow \infty$ ) such that the ensemble average is well converged.

Typical Eulerian-Lagrangian multiphase flow simulations will involve only a finite number of particles per grid cell (finite $M$ ). This will result in a systematic deviation, which is termed the bias error:

$$
\text { Bias Error: } \quad \overline{\bar{e}}_{B}(N, M)=\overline{\bar{e}}-[\overline{\bar{e}}]_{M \rightarrow \infty},
$$

which is a function of both the number of grid cells $(N)$ and the average number of particles per cell $(M)$. Thus, the deterministic error has been separated into two components: bias and discretization errors. The bias error represents the error of using a finite versus infinite number of Lagrangian particles. The discretization error is the error caused by representing the continuous spatial variation in volume fraction with a finite number of Eulerian grid cells.

The mean square error of an individual realization is a superposition of the deterministic and stochastic error components

$$
\bar{e}_{p}=\overline{\bar{e}}_{D}(N)+\overline{\bar{e}}_{B}(N, M)+\bar{e}_{S}(N, M),
$$


where the stochastic or statistical error, $\bar{e}_{S}(N, M)$, is the difference between an individual realization and the ensemble averaged error, $\overline{\bar{e}}$. The ensemble averaged error is the deterministic error, captured by the first two terms on the right hand side. The stochastic error is a random variable of zero mean and its standard deviation is obtained as

$$
\text { Stochastic Error: } \quad \sigma_{S}=\sqrt{\frac{1}{P} \sum_{p=1}^{P}\left[\bar{e}_{p}-\overline{\bar{e}}\right]^{2}} .
$$

Pope[32], Xu and Pope [31] and Garg et al. [18] have demonstrated that with increasing number of Lagrangian particles the standard deviation of the stochastic error goes as $\sigma_{S}=a(N) / \sqrt{M}$. The behavior of the coefficient $a(N)$ can be obtained with the following argument for the special case of uniform volume fraction distribution. In the limit of $\alpha_{1}=0$, it can be argued that $\bar{e}_{p}$ is the sample variance of $N$ binomially distributed random variables (see section 3.1 below), which is itself a random variable. The expected value of this sample variance is $\overline{\bar{e}}$ and from the standard deviation value of the sample variance is obtained as [33]

$$
a(N) \propto \sqrt{\frac{2}{N-1}+\frac{k}{N}}
$$

where $k$ is the excess kurtosis of the underlying binomial distribution. Based on this argument, even for non-uniform volume fraction distribution, the leading order behavior of the stochastic error is expected to go as $\sigma_{S} \sim 1 / \sqrt{N M}$.

Pope[32], Xu and Pope [31] and Garg et al. [18] have also shown the bias error to behave as $b(N) / M$, where the constant $b(N)$ is a function of grid resolution. In the limit of $M \rightarrow \infty$, both the bias and stochastic errors vanish and the only remaining contribution is the discretization error. The discretization error can be modeled as $C / N^{q}$, where the constant $C$ and the exponent $q$ depend on the details and the order of accuracy of the GB or PB method.

\section{Analytical evaluation of error}

For the simplest GB and PB methods, it is possible to analytically calculate the mean squared error and precisely obtain the bias and discretization components. In what follows explicit expressions for these two errors are obtained for the zeroth order GB method and the top-hat PB method. The analytical results will then be compared with those obtained from numerical simulations.

\subsection{Analytical calculation of GB particle volume fraction}

The simplest method of GB particle volume fraction calculation is considered: for each Eulerian grid cell, the number of particles within the cell is summed to determine the particle volume fraction within the cell. This is the zeroth order method discussed in section 2 .

For a particle whose position is randomly chosen within the domain $0 \leq x \leq 2 \pi$ according to the probability distribution given in (3), the probability of it being in cell $i$ is distributed as

$$
p_{i}=\int_{\frac{2 \pi(i-1)}{N}}^{\frac{2 \pi i}{N}} \frac{1}{2 \pi}\left(1+\alpha_{1} \cos (\xi)\right) d \xi=\frac{1}{N}+\frac{\alpha_{1}}{\pi} \cos \left(\frac{2 \pi}{N}\left(i-\frac{1}{2}\right)\right) \sin \left(\frac{\pi}{N}\right) .
$$


Again we emphasize that this probability is valid only in the limit of low volume fraction where a Poisson point process applies. Since the positions of all $N M$ particles are similarly chosen randomly and independently, the binomial distribution applies. The mean number of particles, $\left\langle n_{i}\right\rangle$, and the variance in the number of particles within the cell, $V_{n, i}$, are then given by

$$
\begin{aligned}
\langle n\rangle_{i} & =N M p_{i}=M+\frac{\alpha_{1}}{\pi} N M \cos \left(\frac{2 \pi}{N}\left(i-\frac{1}{2}\right)\right) \sin \left(\frac{\pi}{N}\right) \\
V_{n, i} & =N M p_{i}\left[1-p_{i}\right] \\
& =N M\left\{\left(\frac{1}{N}-\frac{1}{N^{2}}\right)+\frac{\alpha_{1}}{\pi}\left(1-\frac{2}{N}\right) \cos \left(\frac{2 \pi}{N}\left(i-\frac{1}{2}\right)\right) \sin \left(\frac{\pi}{N}\right)-\frac{\alpha_{1}^{2}}{\pi^{2}} \cos ^{2}\left(\frac{2 \pi}{N}\left(i-\frac{1}{2}\right)\right) \sin ^{2}\left(\frac{\pi}{N}\right)\right\}
\end{aligned}
$$

From which the mean and the variance of the particle volume fraction within cell $i$ are calculated as,

$$
\begin{aligned}
& \langle\alpha\rangle_{i}=N \alpha_{\text {avg }} p_{i}=\alpha_{\text {avg }}\left[1+\frac{\alpha_{1}}{\pi} N \cos \left(\frac{2 \pi}{N}\left(i-\frac{1}{2}\right)\right) \sin \left(\frac{\pi}{N}\right)\right], \\
& V_{\alpha, i}=V_{n, i} \frac{\alpha_{\text {avg }}^{2}}{M^{2}} \\
& =N M \alpha_{\text {avg }}^{2}\left\{\left(\frac{N-1}{N^{2}}\right)+\frac{\alpha_{1}}{\pi}\left(1-\frac{2}{N}\right) \cos \left(\frac{2 \pi}{N}\left(i-\frac{1}{2}\right)\right) \sin \left(\frac{\pi}{N}\right)-\frac{\alpha_{1}^{2}}{\pi^{2}} \cos ^{2}\left(\frac{2 \pi}{N}\left(i-\frac{1}{2}\right)\right) \sin ^{2}\left(\frac{\pi}{N}\right)\right\} .
\end{aligned}
$$

The above variances are based on deviation from the respective mean. However, in the error analysis the deviation from $\alpha_{\text {exact }}$ is of interest. Thus, the variance of the calculated particle volume fraction with respect to the exact particle volume fraction within cell $i$ can be obtained as

$$
\operatorname{Var}_{i}=\left\langle\left(\alpha-\alpha_{\text {exact }}(x)\right)^{2}\right\rangle=V_{\alpha, i}+\int_{2 \pi(i-1) / N}^{2 \pi i / N}\left\{\alpha_{\text {exact }}-\langle\alpha\rangle\right\}^{2} d x
$$

The above when summed over all $N$ grid cells will yield the mean square error in the numerical approximation to the particle volume fraction. However, it must be noted that particles are nonuniformly distributed within the domain according to probability (3). By appropriately weighting this non-uniform distribution in the summation over all the grid cells, the mean squared error can be calculated as,

$$
\begin{aligned}
\overline{\bar{e}}_{0}= & \frac{1}{\alpha_{\text {avg }}^{2}} \sum_{i=1}^{N} \operatorname{Var}_{i} \int_{2 \pi(i-1) / N}^{2 \pi i / N} \frac{1}{2 \pi}\left[1+\alpha_{1} \cos (x)\right] d x \\
& =\frac{1}{M}\left(\frac{N-1}{N}\right)+\frac{N^{2}}{M} \frac{\alpha_{1}^{2}}{2 \pi^{2}}\left(1-\frac{2}{N}\right) \sin ^{2}\left(\frac{\pi}{N}\right)-\frac{N}{M} \frac{\alpha_{1}^{2}}{2 \pi^{2}} \sin ^{2}\left(\frac{\pi}{N}\right)+\alpha_{1}^{2}\left[\frac{\pi}{N}-\frac{N}{\pi} \sin ^{2}\left(\frac{\pi}{N}\right)\right] .
\end{aligned}
$$

The first three terms represent the bias error. As expected the mean square bias error decreases as $1 / M$ and goes to zero as $M \rightarrow \infty$. The final term is the discretization error, which is the only remaining error as $M \rightarrow \infty$. By construction, the above mean square error is an average over an ensemble of realizations and thus represented with a double overbar. It does not include the stochastic error. Stochastic error arises when the above ensemble averaged variance is approximated in an individual realization by an average over a finite sample size of particles with a discretization of $N$ grid cells. 
The mean square error is also computed numerically from an ensemble of realizations. In each realization, $N M$ Lagrangian particles are randomly located within the domain following the probability given in (3). The local volume fraction at each particle is then computed with the zeroth order GB method. By comparing the computed value of particle volume fraction against the exact value, and averaging over all particles and over all realizations, discretization and bias errors can be calculated as given in (24) and (25). With increasing ensemble size (i.e., as $P \rightarrow \infty$ ) the bias error is verified to approach the sum of the first three terms of (35) and the discretization error approaches the last term of (35).

In Fig. 1a, the mean square error given in (35) is plotted as a function of grid resolution $N$ for a fixed value of $M=64$ and three different values of $\alpha_{1}$. The corresponding bias and discretization errors are shown in Fig. $1 \mathrm{~b}$ (note: the discretization error for $\alpha_{1}=0.0$ is zero). The behavior of the bias and discretization errors as $N \rightarrow \infty$ are

$$
\overline{\bar{e}}_{B 0, N \rightarrow \infty}=\frac{1}{M}\left(1+\frac{1}{2} \alpha_{1}^{2}\right)-\frac{1}{M N}\left(1+\frac{3}{2} \alpha_{1}^{2}\right) \quad \text { and } \quad \overline{\bar{e}}_{D 0, N \rightarrow \infty}=\frac{1}{3} \frac{\alpha_{1}^{2} \pi^{3}}{N^{3}} .
$$

The bias error is observed to increase with increasing $N$ and to reach an asymptotic value of $(2+$ $\left.\alpha_{1}^{2}\right) / 2 M$. In the limit of uniform volume fraction, the bias error increases by factor 2 from $N=2$ to $N \rightarrow$ $\infty$, and the increase is slightly larger for non-zero $\alpha_{1}$. The discretization error is observed to decay as $N^{-3}$. As a result, for $\alpha_{1}=0$ the mean square error monotonically increases with increasing $N$ (due to bias error), while for $\alpha_{1}=0.5$ the mean square error monotonically decreases with increasing $N$. The decrease in error for small $N$ is due to the dominant contribution from the discretization error, but mean square error reaches the asymptotic value of $1.125 / M$ for large $N$. For intermediate values of $\alpha_{1}$, mean square error decreases for small $N$ due to the dominant contribution from discretization error and then slowly increases for large $N$ to its asymptotic value.

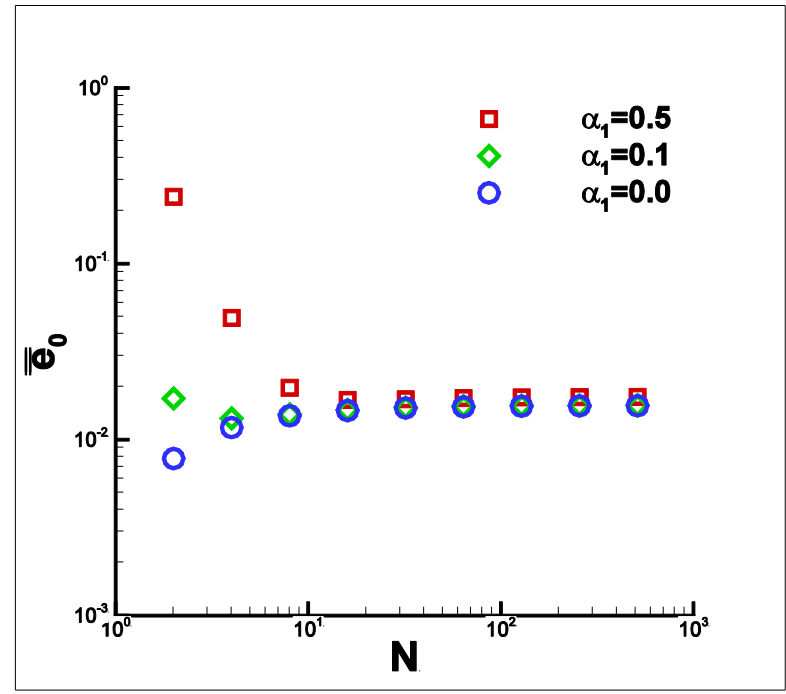

(a)

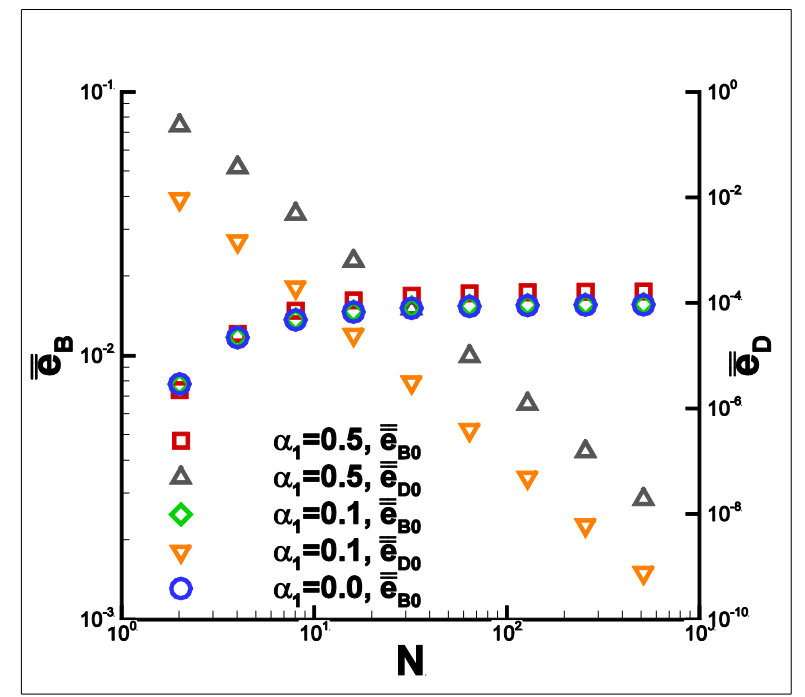

(b)

Fig. 1. Zeroth order GB method, $M=64$, a) analytical mean squared error and b) analytical bias and discretization error.

Using (35), the value of $N$ that minimizes the mean square (deterministic) error for a given value of $N M$ and $\alpha_{1}$ may be found. There is a minimum value of deterministic error for non-zero values of $\alpha_{1}$ because, for a fixed total number of particles, as the number of grid cells increase, the discretization 
error decreases while the bias error increases. Analytically, the optimal value of $N$ may be evaluated from (35). For a fixed, but large, total number of particles per wavelength (i.e., fixed $N M$ ) the value of $N$ which minimizes the deterministic error increases slowly as $(N M)^{1 / 4}$. Thus even for values of $N M \sim O\left(10^{4}\right)$ the optimal number of grid cells per wavelength lies within $2 \leq N \leq 20$. The optimal values of $N$ yielding minimal deterministic error are shown in Fig. 3 for varying values of total number of particles per wave using two different values of $\alpha_{1}$.

\subsection{Analytical calculation of PB particle volume fraction}

Here again the simplest method of PB volume fraction calculation is considered: all surrounding particles in the region $\left[-\frac{\delta}{2}, \frac{\delta}{2}\right]$ are considered and a uniform weight is applied in the calculation of local volume fraction. This method is the top-hat PB method discussed in section 2.

The probability of a particle being in the $\delta$ neighborhood of the reference particle, which is located at $x$, is defined as

$$
p(x)=\int_{x-\delta / 2}^{x+\delta / 2} \frac{1}{2 \pi}\left(1+\alpha_{1} \cos (\xi)\right) d \xi=\frac{\delta}{2 \pi}+\frac{\alpha_{1}}{\pi} \cos (x) \sin \left(\frac{\pi}{N_{p}}\right) .
$$

The mean number of particles within this range, $\langle n\rangle(x)$, and the variance in the number of particles within the range, $V_{n}(x)$, can be obtained from the binomial distribution. From $\langle n\rangle(x)$ and $V_{n}(x)$ the mean volume fraction, $\langle\alpha\rangle(x)$, and the variance in volume fraction, $V_{\alpha}(x)$, is obtained as

$$
\begin{aligned}
& \langle\alpha\rangle(x)=N_{p} \alpha_{\text {avg }} p(x)=\alpha_{\text {avg }}\left[1+\frac{\alpha_{1}}{\pi} N_{p} \cos (x) \sin \left(\frac{\pi}{N_{p}}\right)\right] \\
& V_{\alpha}(x)=\frac{N}{M} \alpha_{\text {avg }}^{2}\left\{\left(\frac{N_{p}-1}{N_{p}^{2}}\right)+\frac{\alpha_{1}}{\pi}\left(1-\frac{2}{N_{p}}\right) \cos (x) \sin \left(\frac{\pi}{N_{p}}\right)-\frac{\alpha_{1}^{2}}{\pi^{2}} \cos ^{2}(x) \sin ^{2}\left(\frac{\pi}{N_{p}}\right)\right\} .
\end{aligned}
$$

Although the top-hat PB method is independent of an Eulerian grid, to facilitate comparison with the analysis of the previous section for the zeroth order GB method, here $N_{p}=2 \pi / \delta$ is defined to be the number of top-hat widths within the domain and $M$ to be the average number of particles within a tophat width. The above variance is based on deviation from $\langle\alpha\rangle(x)$. For the error analysis deviation from the exact volume fraction, $\alpha_{\text {exact }}$ is required. As before, variance of actual particle volume fraction with respect to the exact particle volume fraction can be obtained as

$$
\operatorname{Var}(x)=\left\langle\left(\alpha-\alpha_{\text {exact }}(x)\right)^{2}\right\rangle=V_{\alpha}(x)+\left\{\alpha_{\text {exact }}-\langle\alpha\rangle\right\}^{2} .
$$

The above can be integrated over the domain to obtain the mean squared error as,

$$
\begin{aligned}
\overline{\bar{e}}_{T H} & =\frac{1}{2 \pi} \int_{0}^{2 \pi} \frac{\operatorname{Var}(x)}{\alpha_{\text {avg }}^{2}}\left[1+\alpha_{1} \cos (x)\right] d x \\
& =\frac{1}{M}\left(\frac{N_{p}-1}{N_{p}}\right)+\frac{N_{p}}{M} \frac{\alpha_{1}^{2}}{2 \pi}\left(1-\frac{2}{N_{p}}\right) \sin \left(\frac{\pi}{N_{p}}\right)-\frac{N_{p}}{M} \frac{\alpha_{1}^{2}}{2 \pi^{2}} \sin ^{2}\left(\frac{\pi}{N_{p}}\right)+\frac{\alpha_{1}^{2}}{2}\left[1-\frac{N_{p}}{\pi} \sin \left(\frac{\pi}{N_{p}}\right)\right]^{2} .
\end{aligned}
$$

The first three terms represent the bias error, where bias error goes to zero as $M \rightarrow \infty$. The final term is the discretization error, which is the only remaining term when $M \rightarrow \infty$. The behavior of the bias and discretization errors as $\delta \rightarrow 0$ (i.e., $N_{p} \rightarrow \infty$ ) are 


$$
\overline{\bar{e}}_{B, T H, N_{p} \rightarrow \infty}=\frac{1}{M}\left(1+\frac{1}{2} \alpha_{1}^{2}\right)-\frac{1}{M N_{p}}\left(1+\frac{3}{2} \alpha_{1}^{2}\right) \quad \text { and } \quad \overline{\bar{e}}_{D, T H, N_{p} \rightarrow \infty}=\frac{1}{72} \frac{\alpha_{1}^{2} \pi^{4}}{N_{p}^{4}} .
$$

A plot of $\overline{\bar{e}}_{T H}$ as a function of $N_{p}$ for a fixed value of $M=64$ for three different values of $\alpha_{1}$ is shown in Fig. 2a. As can be seen from (41) the discretization error decays as $N_{p}^{-4}$, faster than the zeroth order GB method. The bias error, as for the zeroth order GB method, increases with $N_{p}$. However in the top-hat PB method for $M=64$ the bias error generally dominates. With increasing $N$, as bias error increases, the bias and discretization errors will intersect and yield an optimum $N_{p}$ at which the sum of bias and discretization errors will be a minimum. In the limit of $N_{p} \rightarrow \infty$ both the zeroth order GB method and the top-hat PB method yield identical errors for all values of $\alpha_{1}$. However, for finite $N_{p}$ the errors from the top-hat PB method are lower than those from the zeroth order GB method, due to lower discretization contribution.

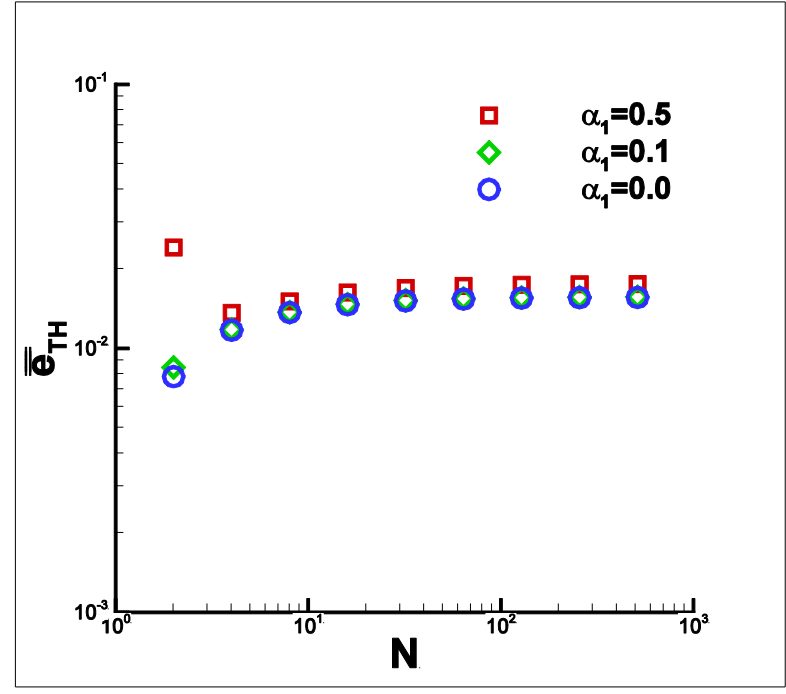

(a)

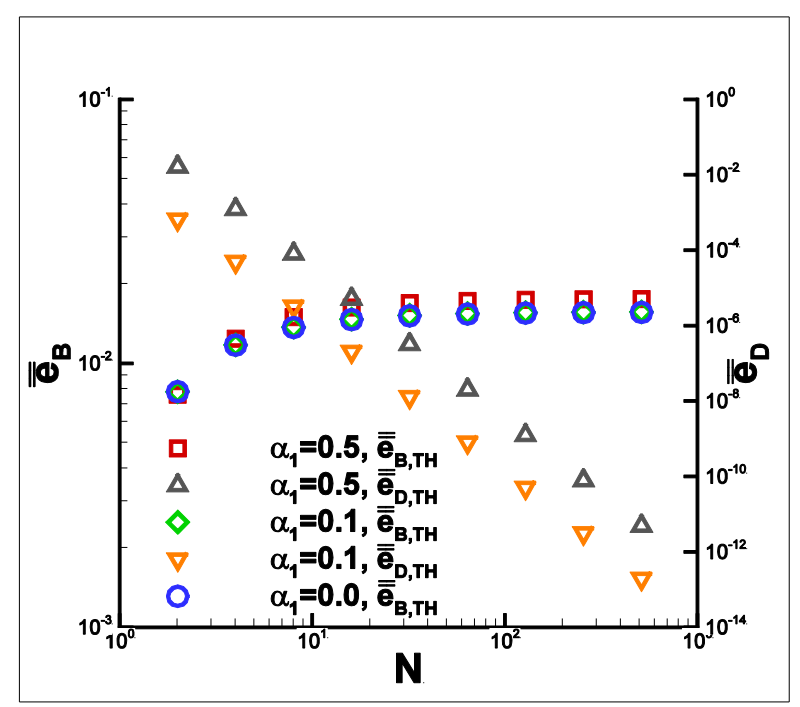

(b)

Fig. 2. Top-hat method, $M=64$, a) analytical mean squared error and b) analytical bias and discretization error.

Similar to the zeroth order GB method, using (41), the value of $N_{p}$ that minimizes the deterministic error may be found for a given value of $N_{p} M$ and $\alpha_{1}$. Again the optimum value of $N_{p}$ increases slowly as $\left(N_{p} M\right)^{1 / 4}$. The optimal value of $N_{p}$ may be solved from (41) and observe it to be in the range $2 \leq N_{p} \leq$ 8 for $N_{p} M \leq O\left(10^{4}\right)$. The optimal value of $N_{p}$ yielding the minimal value of deterministic error are shown in Fig. 3 for varying values of total number of particles per wave using two different values of $\alpha_{1}$.

\subsection{Optimal width for Gaussian PB method}

The Gauss PB method is independent of the Eulerian grid cell size, but does depend on the averaging width $\delta$. As discussed in sections 3.1 and 3.2, it is possible to select an optimal value of Gaussian averaging width to minimize the deterministic error. Because the discretization error is the ensemble mean square error in the dense particle limit, the numerical approximation to volume fraction is computed as,

$$
\alpha_{G, N M \rightarrow \infty}(x)=\int_{-\infty}^{\infty} \frac{\alpha_{a v g}}{\delta \sqrt{\pi}}\left(1+\alpha_{1} \cos (x+\xi)\right) \exp \left(-\frac{\xi^{2}}{\delta^{2}}\right) d \xi
$$




$$
=\alpha_{\text {avg }}\left(1+\alpha_{1} \exp \left(-\frac{\delta^{2}}{4}\right) \cos (x)\right) .
$$

Thus the Gaussian averaging acts as a spectral filter and reduces the amplitude of the sinusoidal variation in volume fraction by the multiplicative factor $\exp \left(-\frac{\delta^{2}}{4}\right)$. From the above, the corresponding discretization error can be computed as

$$
\overline{\bar{e}}_{D, G}=\frac{\alpha_{1}^{2}}{2}\left(1-\exp \left(-\frac{\delta^{2}}{4}\right)\right)^{2} .
$$

In the limit of $\delta \rightarrow 0$, the width of the Gaussian is vanishingly narrow, thus the sinusoidal variation is faithfully recovered. As a result, in this limit, the discretization error becomes zero. As the width of the Gaussian increases, the domain of averaging of the sinusoidal variation becomes broader and the discretization error increases.

Because deterministic error consists of both discretization and bias errors, the effect of the Gaussian width on the bias error must be additionally considered. The bias error will depend on the number of particles that significantly contribute to the Gaussian weighted average. The width of the Gaussian increases with $\delta$ and the central weight of a neighboring particle with zero separation decreases as $1 /(\delta \sqrt{\pi})$. All particles located within a distance of $2.146 \delta$ will contribute with a weight that is at least $1 \%$ of the central weight. The mean number of particles that contribute at this level can then be estimated as $2.146 \mathrm{NM} \delta / \pi$. Thus, the bias error (and the stochastic error) is expected to decrease with increasing number of particles per wave and with increasing width of the Gaussian. The optimal Gaussian width for minimization of deterministic error can be numerically determined for the Gaussian PB method and the results are presented in Fig. 3, where for varying $N M$ the optimal Gaussian width $\delta$ and the minimum deterministic error are plotted for $\alpha_{1}=0.5$ and 0.1 . It can be observed that the minimal deterministic error for the PB methods is lower than for the zeroth order GB method.

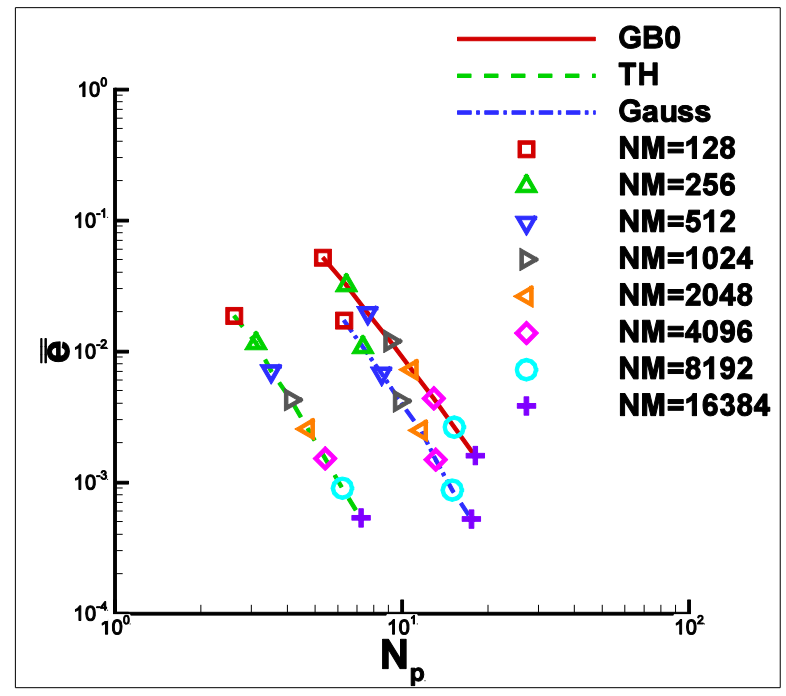

(a)

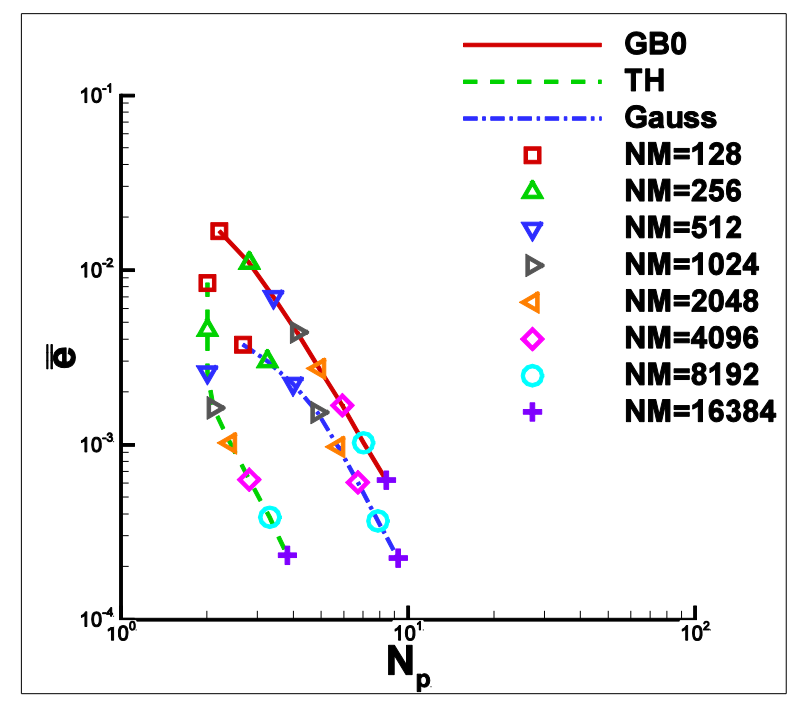

(b)

Fig. 3. Optimal value of $N_{p}$ and corresponding minimum deterministic error for GB zeroth order (GBO), PB top-hat (TH) and PB Gauss methods for varying values of number of particles per wave $(N M)$ for a) $\alpha_{1}=0.5$ and (b) $\alpha_{1}=0.1$. 


\section{Numerical results}

\subsection{Grid-based methods of calculating volume fraction}

\subsubsection{Comparison of exact and numerical solutions of volume fraction}

In the numerical experiments, the periodic domain $0 \leq x \leq 2 \pi$ is divided into $N$ equal grid cells and $N M$ particles are randomly distributed within the domain. The volume fraction at each particle location is calculated using the zeroth, first, and second order GB methods described in section 2. To evaluate the quality of the numerical particle volume fraction the effect of (1) increasing $N$ for constant $M$ and $\alpha_{1}$, (2) increasing $M$ for constant $N$ and $\alpha_{1}$, and (3) decreasing $\alpha_{1}$ for constant $N$ and $M$ are considered. In each case a result from a single realization is presented, and the results for repeated realizations are qualitatively similar.

For $\alpha_{1}=0.5, M=16$, Fig. 4 compares the exact volume fraction at the $N M$ particle locations against the corresponding values obtained from zeroth, first and second order GB methods. The three frames in Fig. 4 are $N=2,8$ and 16 and thereby the effect of increasing $N$ is investigated. For $N=2$ (very few grid cells), as expected, the sinusoidal shape is not well-captured. For $N=16$, the number of grid cells is adequate to represent the overall sinusoidal variation. However, with the increase in the number of grid cells, the probability of an individual particle being placed within a particular cell goes down (recall $p_{i}$ goes as $1 / N$ in equation 29). Correspondingly, variance increases, which leads to more fluctuations (oscillations) in the volume fraction variation. Effectively, the discretization error dominates in Fig. 4a, while the bias and the stochastic errors are large in Fig. 4c.

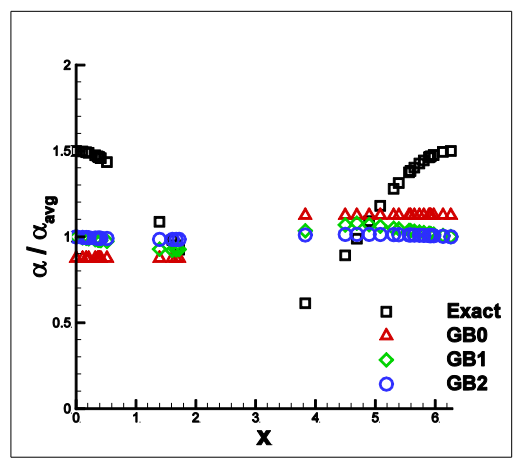

(a)

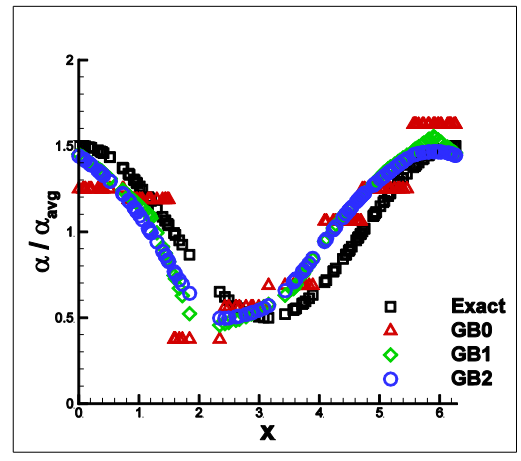

(b)

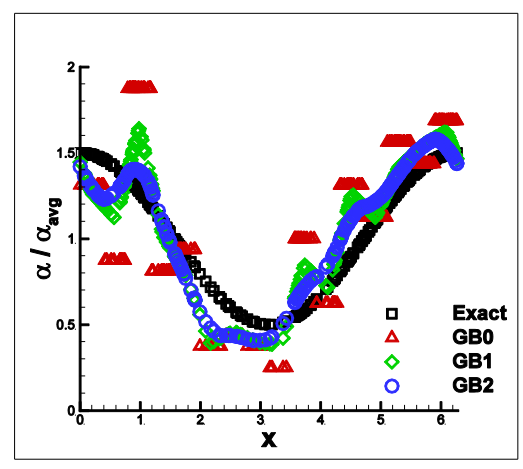

(c)

Fig. 4. Particle volume fraction obtained using the zeroth (GBO), first (GB1) and second (GB2) order GB methods compared to exact volume fraction at $N M$ particle locations for $M=16, \alpha_{1}=0.5$. Frame (a) $N=2$, (b) $N=8$, and (c) $N=16$.

For $\alpha_{1}=0.5, N=8$, Fig. 5 shows the effect of increasing $M$. Since the number of grid cells (and the averaging width) is constant in these plots, the increase in the average number of particles within each cell reduces the bias and the stochastic errors. Thus, as $M$ increases from 4 to 16 and 32 the $\mathrm{GB}$ methods' volume fraction estimation is smoother and approaches the true volume fraction variation. 


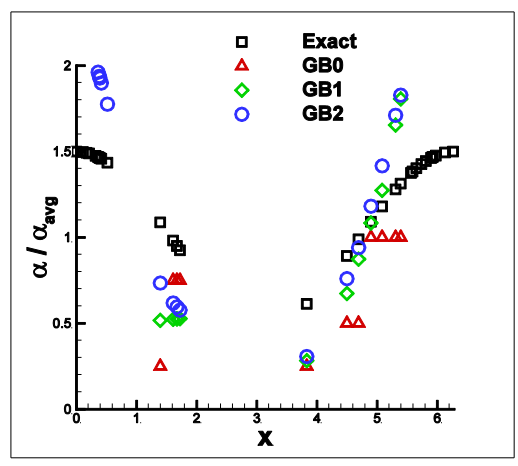

(a)

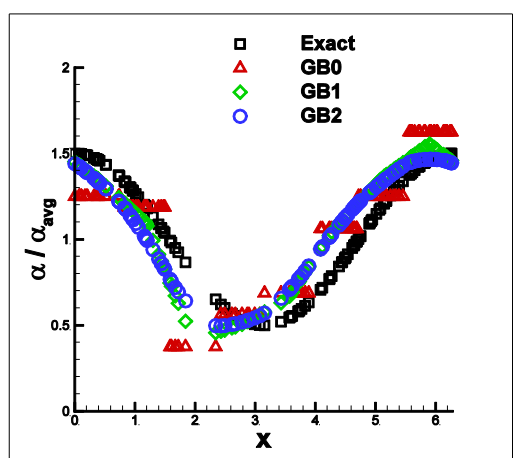

(b)

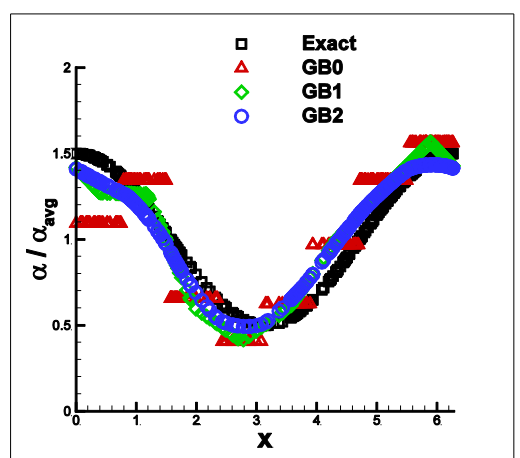

(c)

Fig. 5. Particle volume fraction obtained using the zeroth (GB0), first (GB1) and second (GB2) order GB methods compared to exact volume fraction at $N M$ particle locations for $N=8, \alpha_{1}=0.5$. Frame (a) $M=4$, (b) $M=16$, (c) $M=32$.

Finally, for $N=8, M=16$, Fig. 6 shows the effect of decreasing $\alpha_{1}$. The relative magnitude of the sinusoidal variation in volume fraction decreases (recall equation 1 ) with $\alpha_{1}$. For $\alpha_{1}=0.5$ increasing $N$ beyond an optimal value for constant $M$ leads to a more oscillatory result, and increasing $M$ for a constant $N$ leads to a smoother result. These conclusions hold for $\alpha_{1}=0.0,0.1$ as well. From the analytical error estimate obtained in (35) the level of deviation from the true volume fraction is expected to increase with $\alpha_{1}$. But this increase is quite weak and hard to discern in Fig. 6.

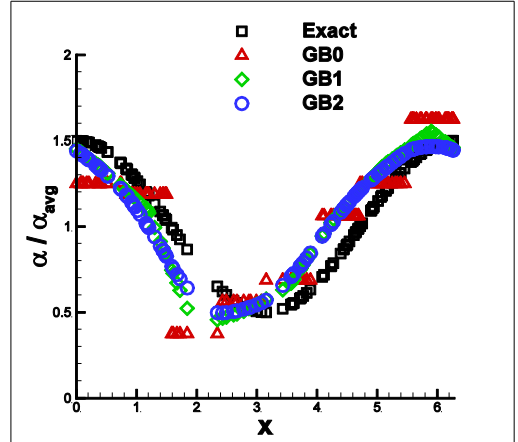

(a)

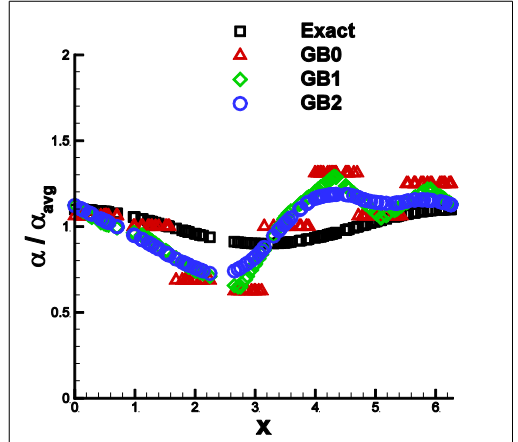

(b)

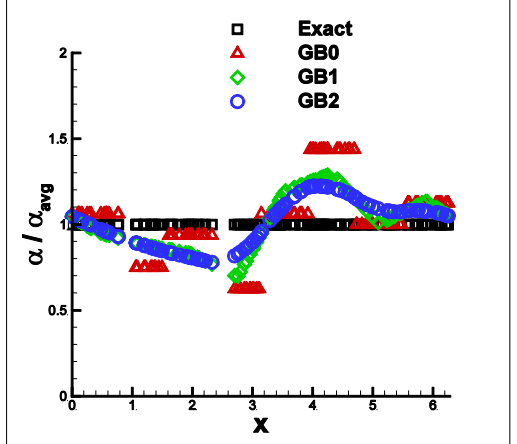

(c)

Fig. 6. Particle volume fraction obtained using the zeroth (GB0), first (GB1) and second (GB2) order GB methods compared to exact volume fraction at $N M$ particle locations for $N=8, M=16$. Frame (a) $\alpha_{1}=0.5$, (b) $\alpha_{1}=0.1$, and (c) $\alpha_{1}=0.0$.

\subsubsection{Convergence of ensemble average}

In the GB methods the particle volume fraction is first calculated for each Eulerian grid cell and then projected to each particle location. By comparing the numerical result to the exact value of volume fraction, the error at each particle location can be calculated. The overall error of an individual realization is then obtained by summing over all the particles to create a mean squared error as given in equation (22).

Fig. 7a shows the plots of $\bar{e}_{p}$ vs the realization number for an ensemble of 5000 realizations (for $\alpha_{1}=$ $0.5, N=8, M=16$ ). The mean square error varies between realizations, but the mean is relatively constant over the ensemble of realizations. The first $P$ realizations are used to calculate the ensemble averaged mean square error (i.e., $\overline{\bar{e}}$ ). A plot of $\overline{\bar{e}}$ vs $P$ is shown in Fig. 7b. The convergence is slow and the 
approach to the true ensemble average is $O(1 / \sqrt{P})$. It is clear from the figure that an average over about 5000 realizations can be considered to approximate the true ensemble average with reasonable accuracy. However, some small fluctuations can still be observed. Results similar to those presented in Fig. 7 were obtained for other values of $\alpha_{1}, N$ and $M$. In all cases 5000 realizations are sufficient for ensemble average, and henceforth ensemble averages are over 5000 realizations.

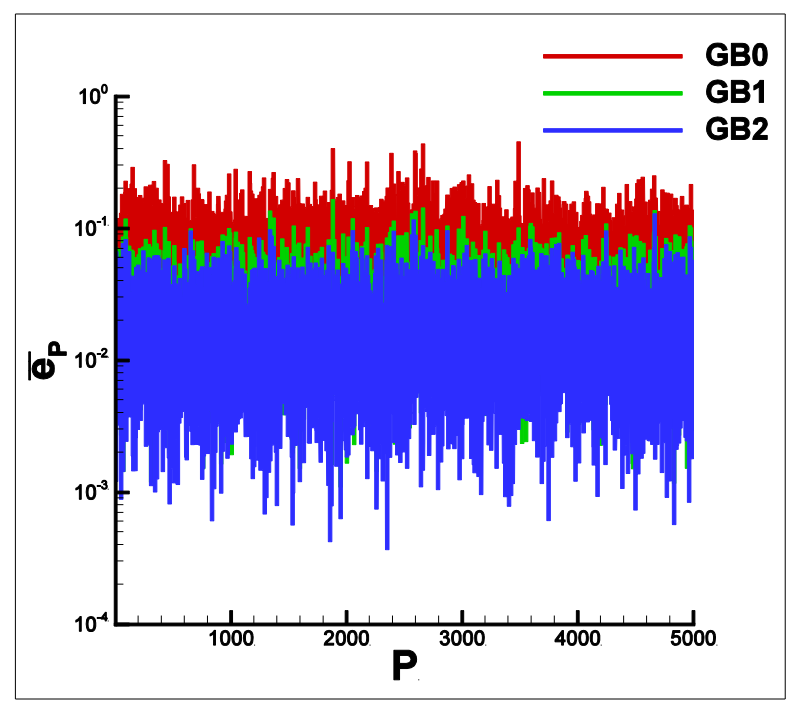

(a)

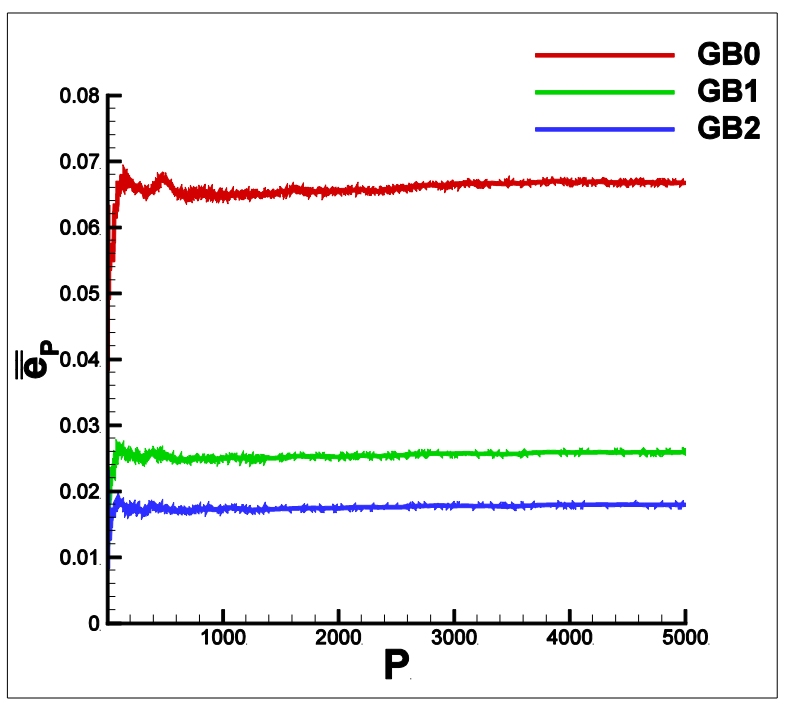

(b)

Fig. 7. (a) Mean Squared Error for 5000 realizations for $\alpha_{1}=0.5, N=8, M=16$ for the zeroth (GB0), first (GB1) and second (GB2) order GB methods of volume fraction evaluation. (b) Ensemble average computed with the first $P$ realizations.

\subsubsection{Discretization, bias, and stochastic errors}

The discretization error is the mean square deviation between the numerical and exact volume fraction that exists as $M \rightarrow \infty$. An analytical expression for the discretization error is available for the zeroth order GB method. For the other methods, the discretization error is computed by plotting the ensemble averaged mean square error $(\overline{\bar{e}})$ as a function of $1 / M$ for varying values of $M$ and extrapolating to the $M \rightarrow \infty$ limit. The resulting discretization error for the zeroth, first, and second order GB methods for $\alpha_{1}=0.5$ are presented in Fig. 8 . 


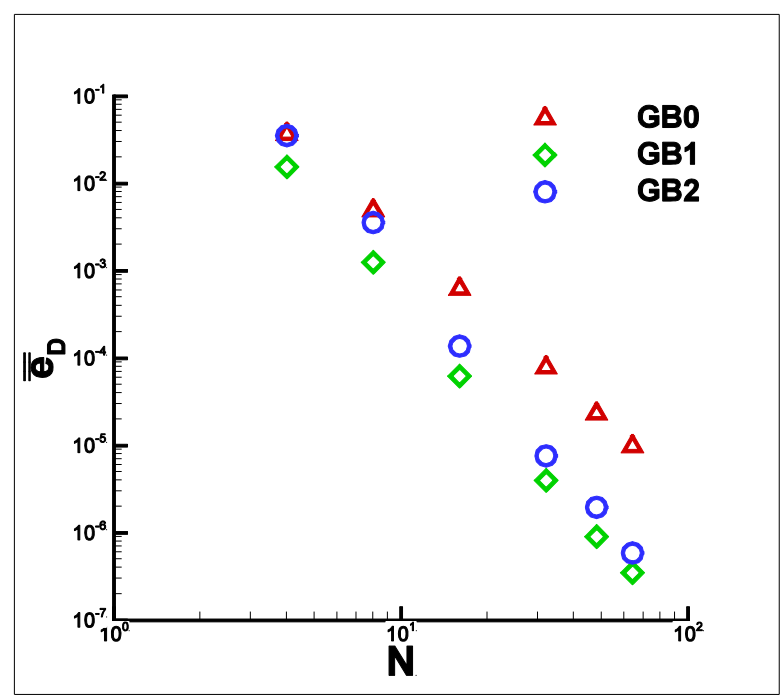

Fig. 8. Discretization error for the zeroth (GB0), first (GB1) and second (GB2) order $\mathrm{GB}$ methods, $\alpha_{1}=0.5$.

The bias error is evaluated from the ensemble averaged mean square error for finite values of $M$ and the corresponding $M \rightarrow \infty$ extrapolation. For the zeroth order GB method the bias error is plotted as a function $1 / M$ in Fig. 9a for two different values of $N$. Numerical and analytical results are shown and compare well. Finally, in Fig. $9 \mathrm{~b}$ the numerical values of the bias error for $\alpha_{1}=0.5$ are shown for the zeroth, first, and second order GB methods. As expected, all methods show an $O(1 / M)$ decay of the bias error with increasing $M$, and the higher order methods show smaller error.

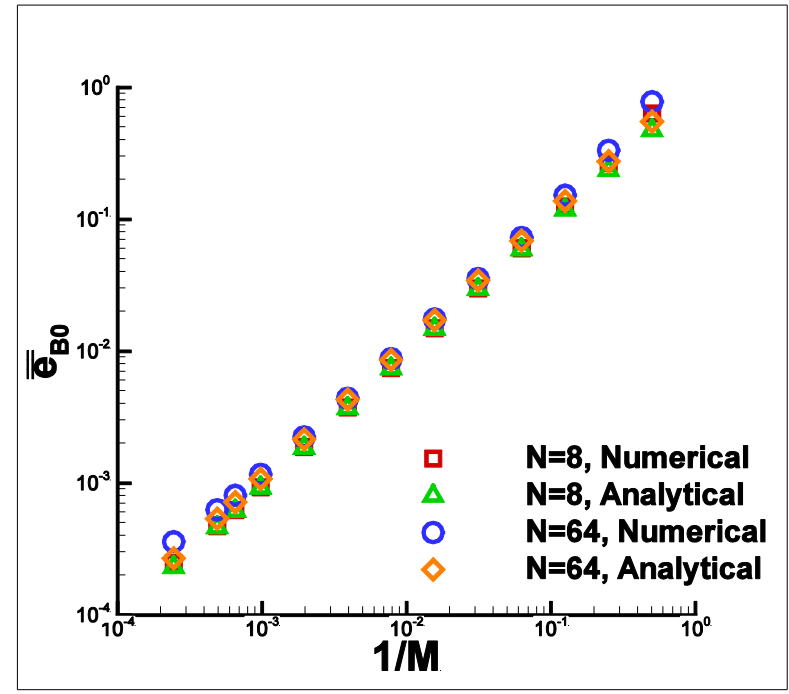

(a)

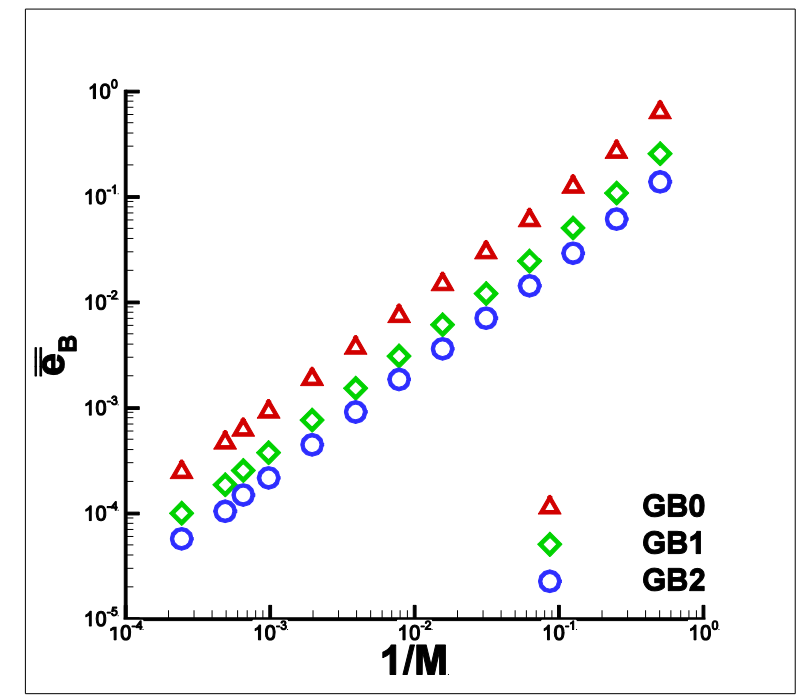

(b)

Fig. 9. Bias error for the a) zeroth order GB method, comparison of analytical and numerical values for $N=8$ and $N=64$, $\left.\alpha_{1}=0.5, \mathrm{~b}\right)$ zeroth (GB0), first (GB1), and second (GB2) order GB methods, $N=8, \alpha_{1}=0.5$.

The stochastic error is evaluated for each realization in (26). Stochastic error may be quantified for a given set of $N, M$ using the mean square difference in the realization mean square error and the ensemble averaged mean square error. For the zeroth, first, and second order GB methods, the stochastic error is plotted as a function of $1 / \sqrt{M}$ in Fig. 10 for two values of $N$. As expected, the higher 
order methods show smaller error, until the saturation point is reached. The stochastic error saturates at large $M$ values (shown for $N=8$ ), where the value of $M$ required for saturation increases with $N$.

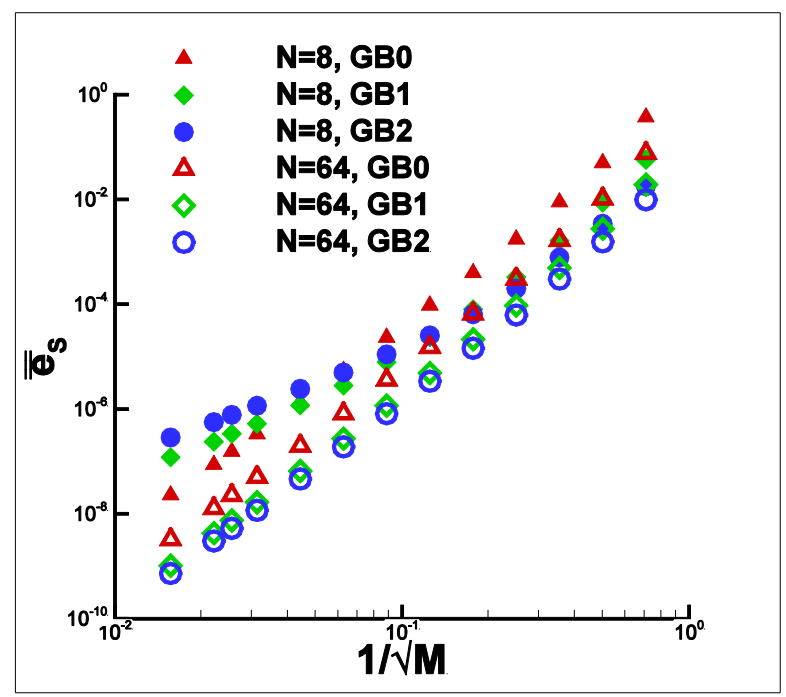

Fig. 10. Stochastic error for the zeroth (GB0), first (GB1), and second (GB2) order GB methods for $N=8$ and $N=64, \alpha_{1}=$ 0.5 .

\subsection{Particle-based methods of calculating volume fraction}

Like GB methods, the PB methods are highly dependent on the width over which the neighboring particles are weighted and averaged in the calculation of the local volume fraction. For GB methods, the width was dependent on the Eulerian grid cell size and the oscillations were observed to increase with increasing number of grid cells (for a constant $M$ in Fig. 4c). PB top-hat and Gaussian methods also require a definition of width over which averaging is performed. It is of interest to investigate the effect of this width on the numerically evaluated particle volume fraction.

The top-hat method discussed in section 2 considers all particles within a region $[-\delta / 2, \delta / 2]$ around the reference particle; in application of the method $\delta$ can be specified as a value independent of the underlying Eulerian grid cell size used for the continuous phase calculation. However, for proper comparison with the GB methods it is convenient to consider $\delta$ to be the same as the specified Eulerian grid cell size (i.e. $\delta=\Delta X=2 \pi / N$ ). The results to be presented below are with this choice of top-hat filter width. The Gaussian method uses the optimal width $\delta$ presented in Fig. 3.

\subsubsection{Comparison of exact and numerical solutions of volume fraction}

As for the GB methods, NM particles are distributed randomly throughout the domain using the probability function described in equation (3). The volume fraction using the PB methods are then numerically calculated. Fig. 11 compares the particle volume fraction estimate for the top-hat and Gaussian PB methods for $N M=256,512,1024$ and $\alpha_{1}=0.5$ (frames a to frame c) and for varying $\alpha_{1}$ for $N M=256$ (frame $d$ to frame f). As the total number of particles is increased, both PB methods show more accurate estimation of the exact volume fraction. The volume fraction estimate using the Gaussian PB method improves as the exact volume fraction transitions from highly sinusoidal to a constant (frame d to frame f). 


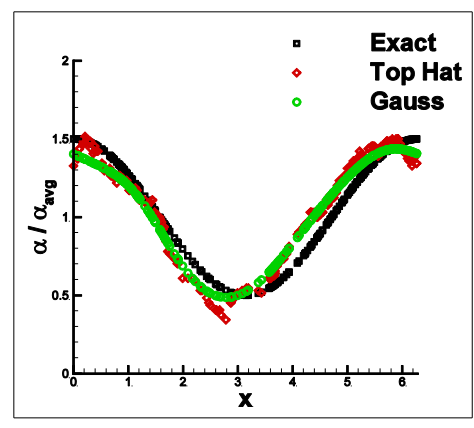

(a)

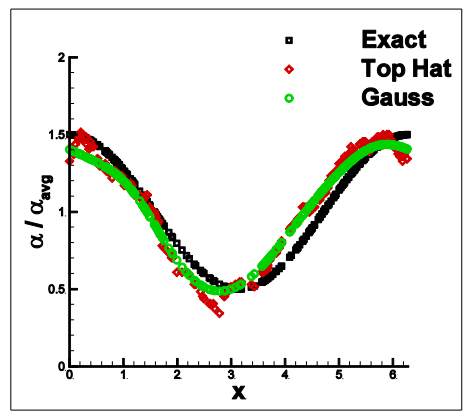

(d)

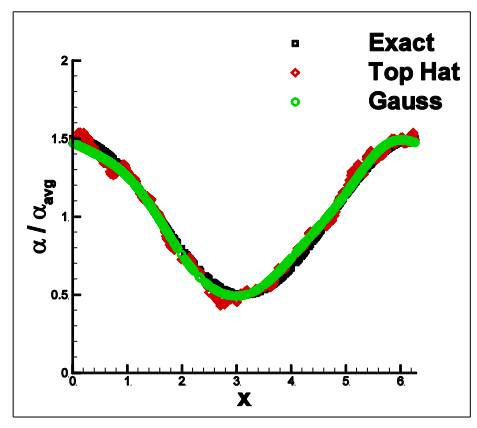

(b)

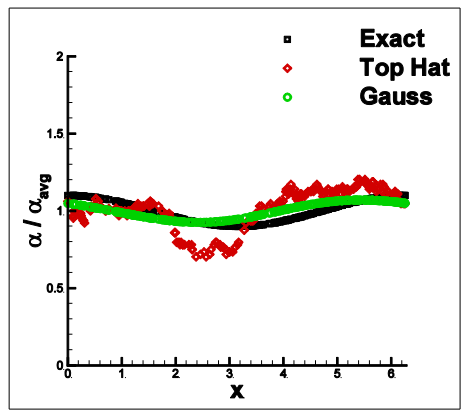

(e)

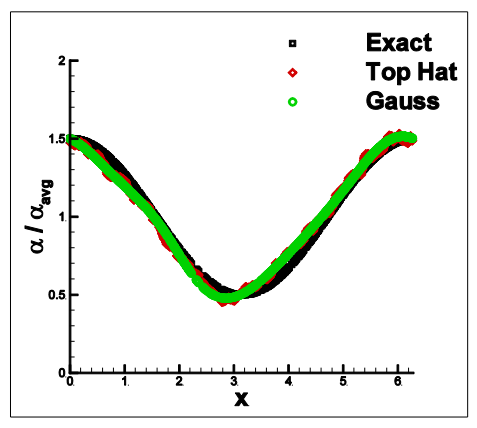

(c)

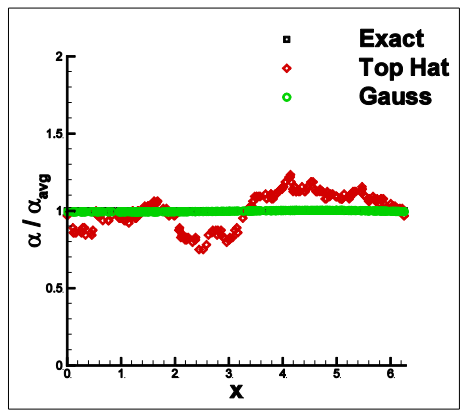

(f)

Fig. 11. Comparison of top-hat PB method (with $N=4$ ) and Gaussian PB method against the exact volume fraction at $\alpha_{1}=$ 0.5 for a) $N M=256$, b) $N M=512$, c) $N M=1024$ and for a fixed value of $N M=256$ for d) $\alpha_{1}=0.5$, e) $\alpha_{1}=0.1$, f) $\alpha_{1}=0.0$.

For both the top-hat and Gaussian PB methods, plots of $\bar{e}_{p}$ vs realization number for an ensemble of several thousand realizations were qualitatively similar to those presented in figure $6 a$ (and thus not shown here). The PB methods were observed to converge faster than the GB methods in general. Thus all ensemble averaged results to be presented here are based on an ensemble of 5000 random realizations

\subsubsection{Deterministic and stochastic errors}

For the top-hat PB method, the analytical results of section 3.2 apply. From (41), since the top-hat filter width is $\delta=2 \pi / N_{p}$, it can be readily seen that the discretization error for the top-hat PB method decays rapidly as $\delta^{4}$. The corresponding bias error is given by first three terms of (41). For the Gaussian PB method, since there is no dependence on an underlying Eulerian grid, the separation of deterministic error into discretization and bias errors is not relevant.

In Fig. 12, the deterministic error (combination of discretization and bias errors) is plotted for the tophat and Gaussian PB methods as a function of the total number of particles and investigate the effect of increasing $\alpha_{1}$. As expected, for all values of $\alpha_{1}$, the deterministic error decreases as the total number of particles increases. For the constant volume fraction case $\left(\alpha_{1}=0.0\right)$ for both PB methods, the deterministic error is dominated by the bias error, which decreases with increasing number of particles. The Gaussian PB method has an averaging width independent of the grid size, thus for constant volume fraction, the deterministic error is orders of magnitude less than the top-hat method, which has been 
bounded to an effective Eulerian grid in the current investigation. For a slight sinusoidal variation of volume fraction ( $\alpha_{1}=0.1$ ), the error is still dominated by the bias error component, yielding a reduction in the deterministic error with increasing number of particles. The effect of the discretization error on the Gaussian PB method becomes apparent. As predicted in (44), the deterministic error is zero for $\alpha_{1}=0.0$, but non-zero when $\alpha_{1}$ is non-zero. For the strong sinusoidal case $\left(\alpha_{1}=0.5\right)$, the error in both PB methods is dominated by bias error for small $N M$, which decreases with the introduction of more particles. Near $N M=10000$, the discretization error begins to dominate the top-hat method (where $N=4$ is held constant), and the deterministic error begins to level off, even as additional particles are added.

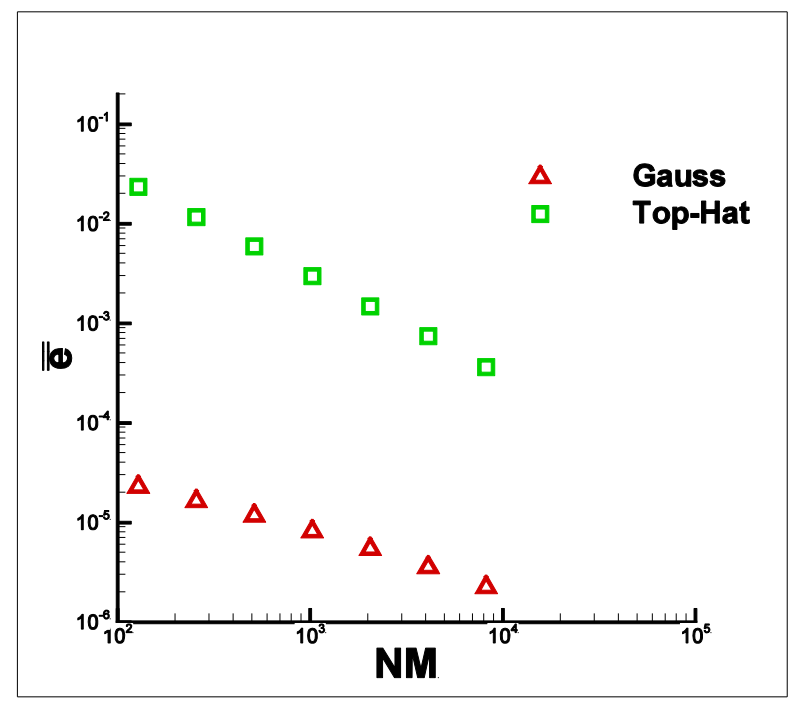

(a)

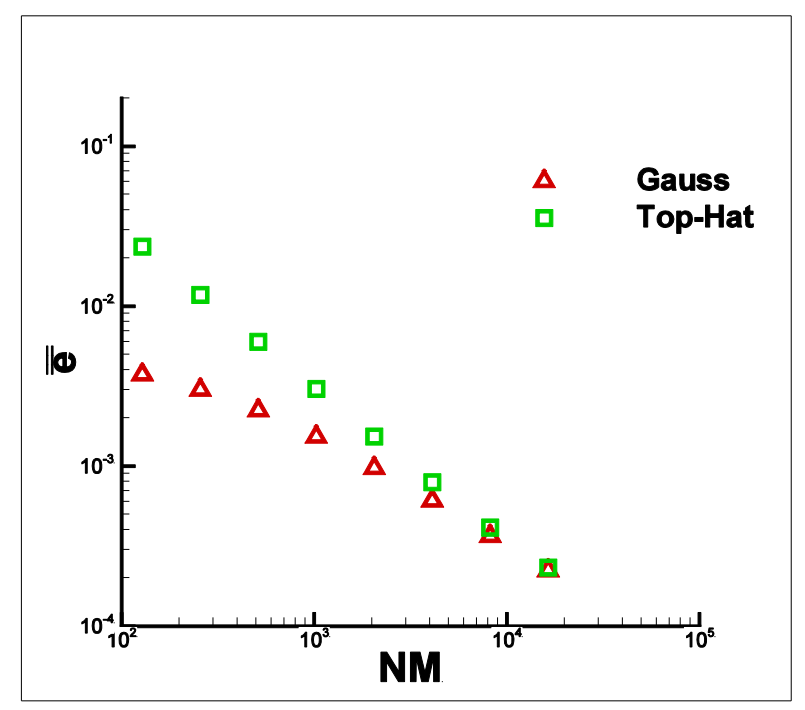

(b)

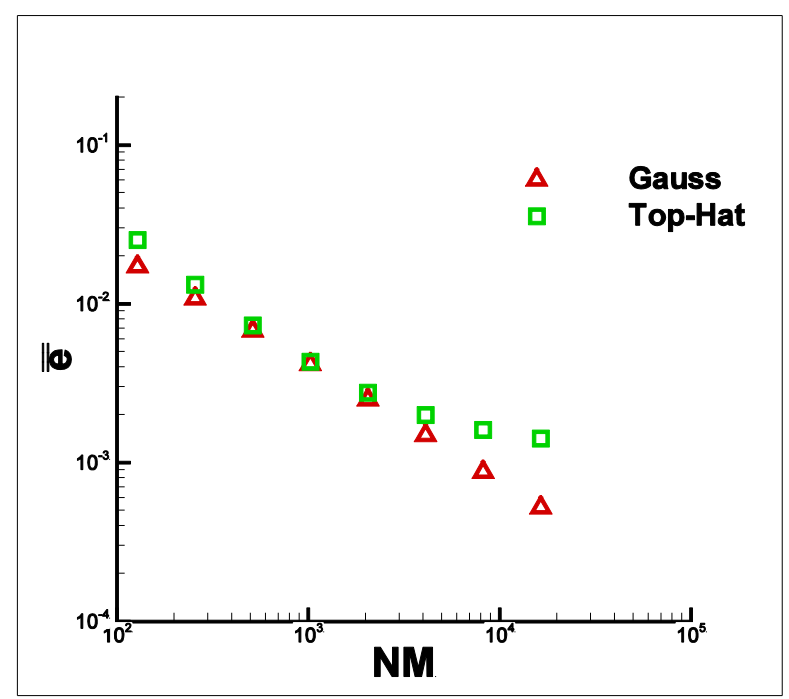

(c)

Fig. 12. Deterministic error for PB methods ( $N=4$ for top-hat), a) $\alpha_{1}=0.0$, b) $\alpha_{1}=0.1$, c) $\alpha_{1}=0.5$. 
For top-hat and Gauss PB methods, the stochastic error is calculated using (27) and the results are plotted as a function of $1 / \sqrt{M}$ in Fig. 13 for two different values of $N$. As expected, all methods show an $O(1 / \sqrt{M})$ decay of the stochastic error with increasing $M$.

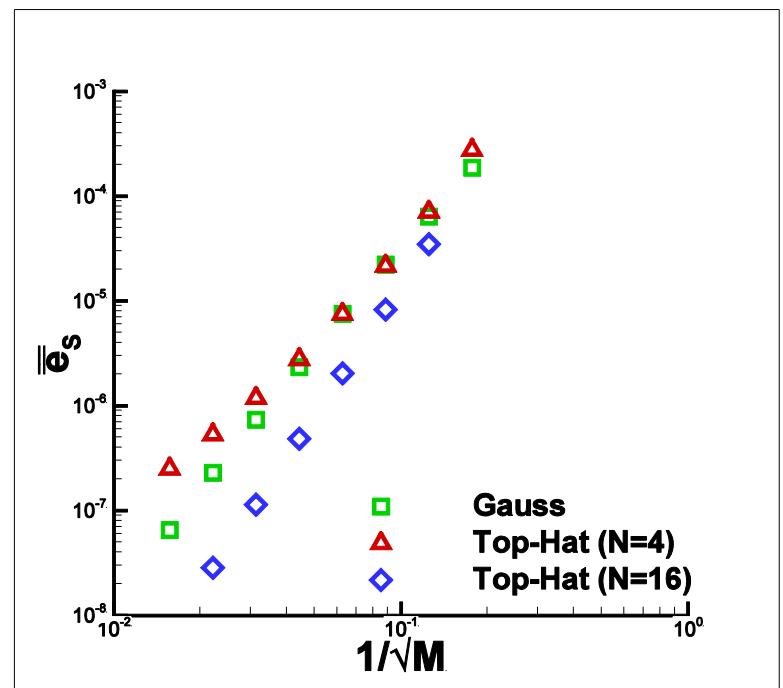

Fig. 13. Stochastic Error for Gauss and top-hat $(N=4, N=16)$ PB methods, $\alpha_{1}=0.5$.

\subsection{Comparison of $\mathrm{GB}$ and $\mathrm{PB}$ methods}

The GB and PB methods may be directly compared by plotting the deterministic error as a function of the total number of particles per wave. For the domain that is minimally discretized $(N=4)$, Fig. 14a, Fig. $14 \mathrm{c}$ and Fig. 14e show the comparison of GB and PB methods. For constant volume fraction $\left(\alpha_{1}=\right.$ 0.0 ), the GB and top-hat PB methods show similar mean squared error and the Gaussian PB method shows significantly reduced error for the same number of particles. As the amplitude of the sinusoidal component of the volume fraction variation $\left(\alpha_{1}=0.5\right)$ is increased, the errors for the GB and PB methods are similar, though a leveling off of the error in all the GB methods and the top-hat PB method is observed, whereas for the Gaussian PB method the error continues to decrease as additional particles are added.

The leveling off of the GB methods and the top-hat PB method are due to large discretization error. For a fixed $N$ as more total particles $(N M)$ are added, $M$ increases. The number of grid cells determines the discretization error, and the number of particles per grid cell determines the bias errors. In Fig. $14 \mathrm{~N}$ is fixed. Thus as $M$ increases, the bias errors decrease. The deterministic error "levels off" when the bias error has decreased sufficiently such that the discretization error dominates. Fig. 2 shows the interplay between the bias and discretization errors.

When the domain is significantly subdivided $(N=16)$ the deterministic error values for the Gaussian PB method is clearly lower than the GB methods for moderate NM values, as shown in Fig. 14b, Fig. 14d, and Fig. 14f. For larger sinusoidal values $\left(\alpha_{1}=0.5\right)$, the GB methods will not level off until much higher $N M$ values.

Note in Fig. 14 the Gaussian PB method is not shown for small $N M$ values. The Gaussian PB method is shown using the optimal $N_{p}$ value for minimum deterministic error. As discussed, for a fixed $N M$ as $N$ increases, the discretization error decreases and the bias error increases. To find the minimal 
deterministic error there must be a local minimum of the bias and discretization errors. For small $N M$, there is no local minimum value.

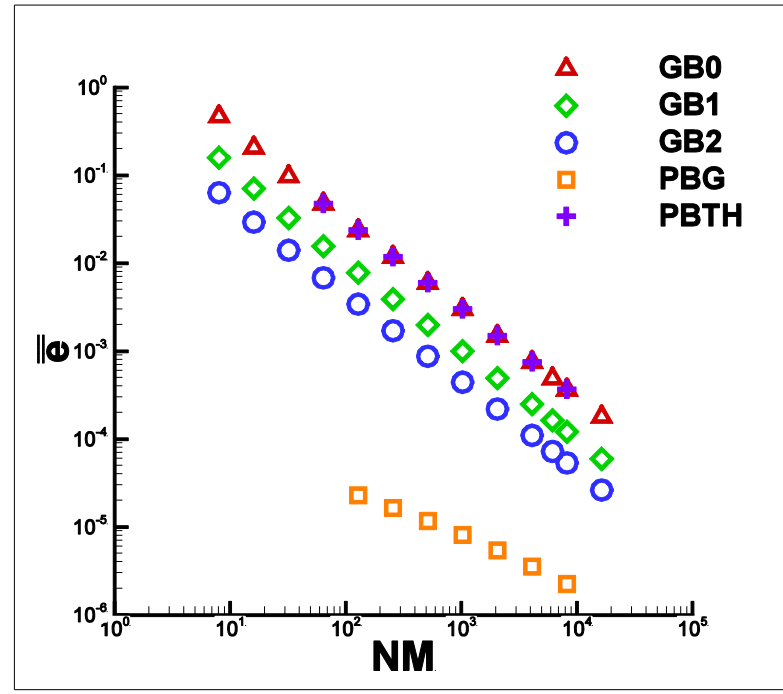

(a)

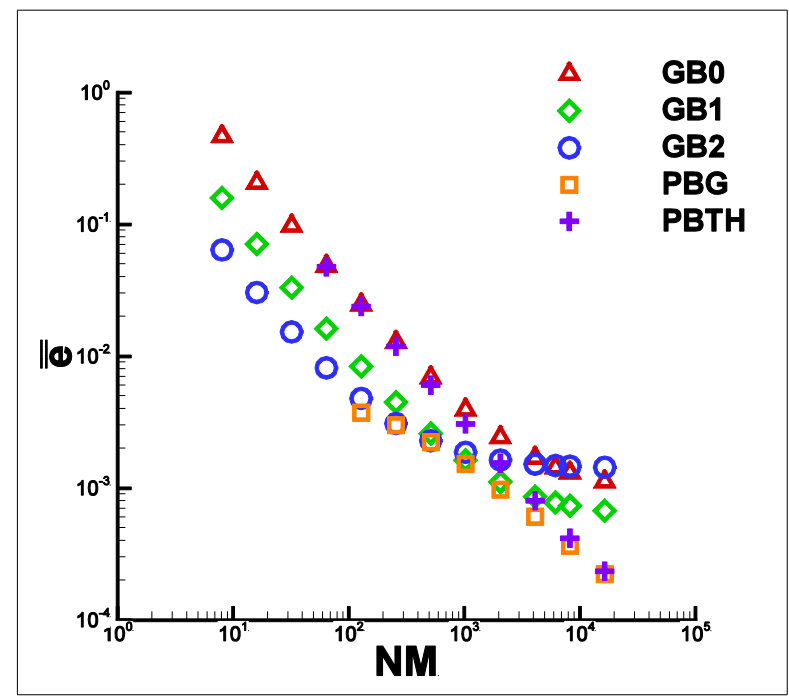

(c)

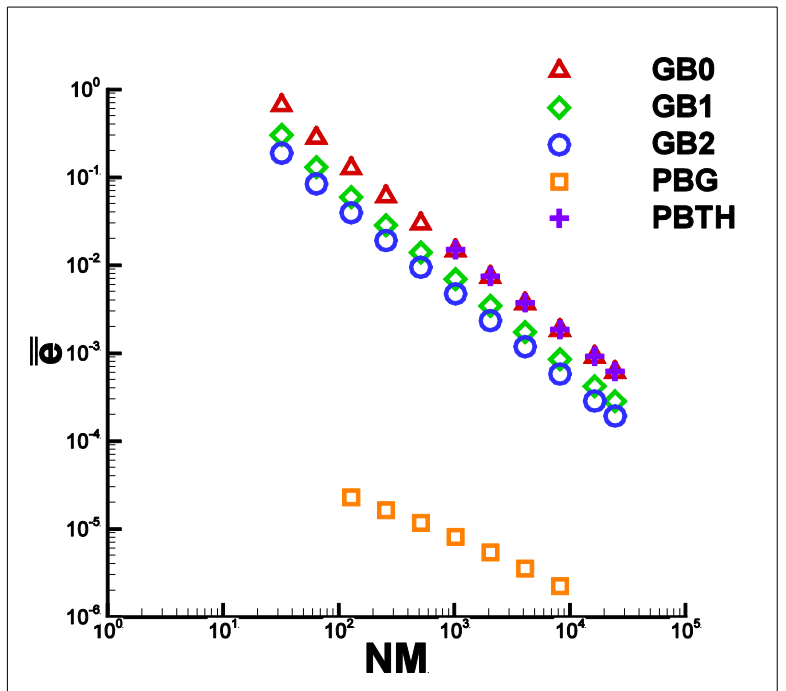

(b)

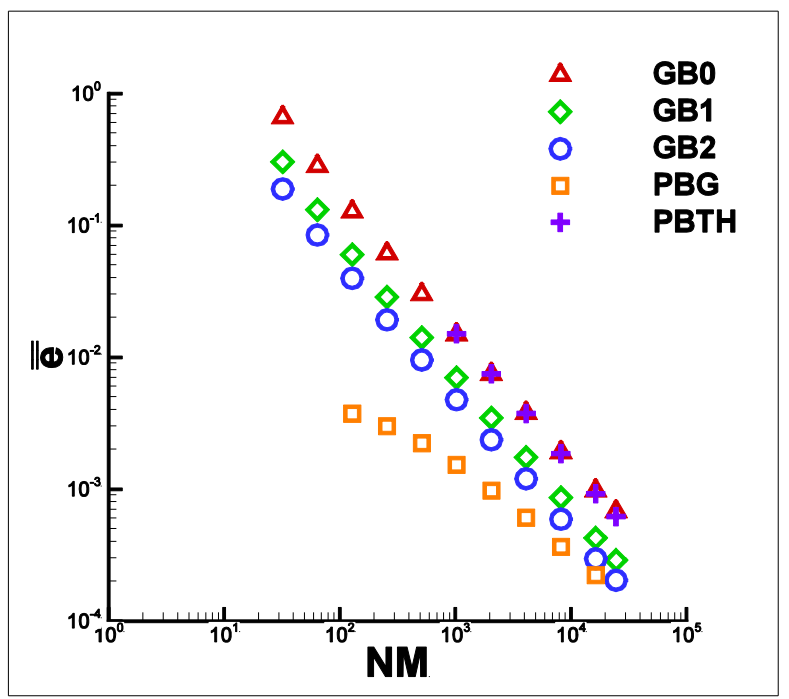

(d) 


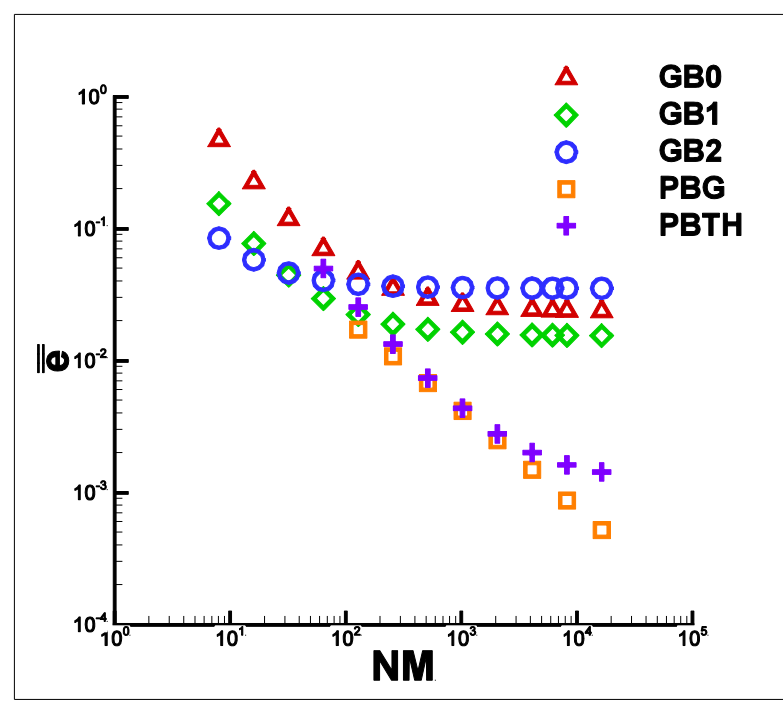

(e)

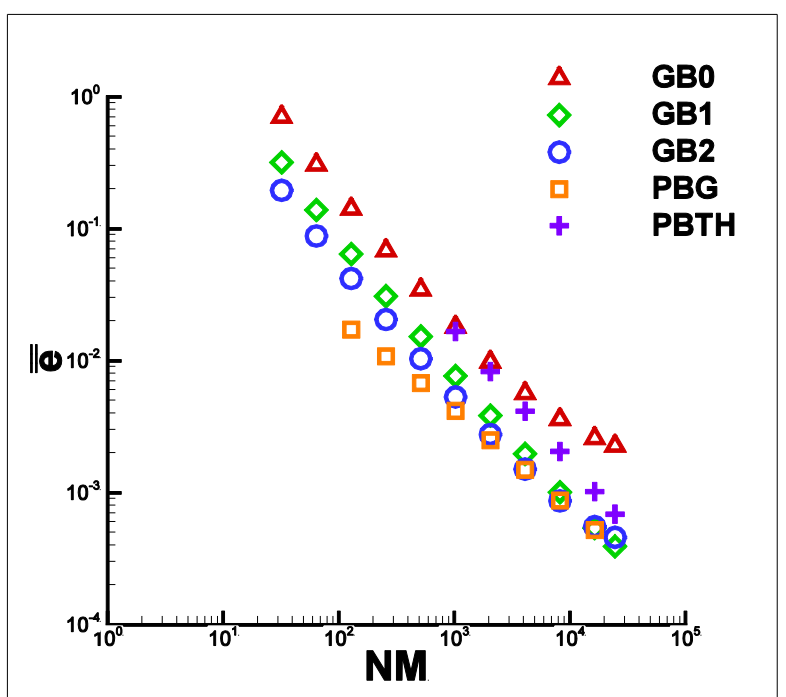

(f)

Fig. 14. A comparison of deterministic error vs total number of particles per wave for the GB and PB methods for varying grid resolution and amplitude of sinusoidal volume fraction variation a) $N=4, \alpha_{1}=0.0$ b) $N=16, \alpha_{1}=0.0$ c) $N=4, \alpha_{1}=$ 0.1 d) $N=16, \alpha_{1}=0.1$ e) $N=4, \alpha_{1}=0.5$ f) $N=16, \alpha_{1}=0.5$.

\section{Discussion}

Now consider the implication of the above results in the case of an Eulerian-Lagrangian simulation of a complex multiphase flow with a range of length and time scales. Restricting attention to only one dimension, let the domain be periodic of length $L_{x}=2 \pi$ and discretized with $N$ Eulerian grid cells. Let the actual particle volume fraction variation within the domain be band-limited and represented by a superposition of Fourier modes of wavelength $2 \pi, \pi, \ldots 2 \pi / j, \ldots, 2 \pi / J$. If $N M$ random Lagrangian particles are distributed within the domain to resolve this volume fraction variation, the question is: how is the resulting error estimated when GB or PB methods are used to numerically approximate the particle volume fraction variation?

Let first consider application of the GB methods. Fourier modes of wavelength shorter than twice the grid cell width (i.e., modes for which $j>N / 2$ ) should be considered sub-grid. Since they violate Nyquist criterion, they will not be well approximated by the GB methods and their error can be expected to be large - this scenario will be discussed in section 5.1. Fourier modes of wavelength longer than twice the grid cell width (i.e., for $j \leq N / 2$ ) are within grid resolution. However, the results of section 4 are directly applicable only for the longest wavelength (i.e., for $j=1$ ) since the error analysis of section 4 was on the basis of $N M$ Lagrangian particles randomly distributed within a single wave.

The theoretical analysis of section 3.1 can be extended to the following volume fraction variation

$$
\alpha_{\text {exact }}(x)=\alpha_{\text {avg }}\left(1+\alpha_{1} \cos [j x]\right),
$$

consisting of $j$ waves. The mean squared error for the zeroth order GB method can now be calculated as,

$$
\overline{\bar{e}}_{0}=\frac{1}{M}\left(\frac{N-1}{N}\right)+\frac{N^{2}}{j^{2} M} \frac{\alpha_{1}^{2}}{2 \pi^{2}}\left(1-\frac{2}{N}\right) \sin ^{2}\left(\frac{\pi j}{N}\right)-\frac{N}{j^{2} M} \frac{\alpha_{1}^{2}}{2 \pi^{2}} \sin ^{2}\left(\frac{\pi j}{N}\right)+\alpha_{1}^{2}\left[\frac{\pi}{N}-\frac{N}{\pi j^{2}} \sin ^{2}\left(\frac{\pi j}{N}\right)\right] .
$$


The first three terms again represent the bias error. As expected the bias error decreases as $1 / M$ and goes to zero as $M \rightarrow \infty$. The final term is the discretization error, which remains as $M \rightarrow \infty$. The long and short wavelength limits can now be explored by taking appropriate limits of the ratio $N / j$, which is the number of grid points per wave.

In the long wavelength limit (i.e., small values of $j$ ) the number of grid cells per wave, $N / j$, will be large and the limiting expressions for the bias and discretization errors can be obtained as

$$
\overline{\bar{e}}_{B 0, N \rightarrow \infty}=\frac{1}{M}\left(1+\frac{1}{2} \alpha_{1}^{2}\right)-\frac{1}{M N}\left(1+\frac{3}{2} \alpha_{1}^{2}\right) \quad \text { and } \quad \overline{\bar{e}}_{D 0, N \rightarrow \infty}=\frac{1}{3} \frac{\alpha_{1}^{2} \pi^{3} j^{2}}{N^{3}} .
$$

The bias error, consistent with (36), is independent of the mode number $j$. The discretization error, as expected, depends on the number of grid cells per wave. As noted in section 3 , for the single mode case of $j=1$, there exists an optimal partitioning of the total number of particles $(N M)$ into the number of grid cells $(N)$ and the average number of particles per cell $(M)$ which minimized the deterministic error, since bias error increases with $N$ while discretization error decreases with $N$. However, in the case of more complex multi-modal volume fraction variation (i.e., J $\gg 1$ ), the total number of grid cells $(N)$ may need to be much larger than the optimal value in order to have sufficient number of grid cells resolving the short wavelength modes. Thus, in the multi-modal context, the discretization error for the long wavelength component of the volume fraction variation will be dominated by the first term of the bias error.

On the other hand, in the short wavelength limit of $j=N / 2,(46)$ becomes

$$
\overline{\bar{e}}_{B 0, N \rightarrow \infty}=\frac{1}{M}\left(1+\frac{2}{\pi^{2}} \alpha_{1}^{2}\right)-\frac{1}{M N}\left(1+\frac{6}{\pi^{2}} \alpha_{1}^{2}\right) \quad \text { and } \quad \overline{\bar{e}}_{D 0, N \rightarrow \infty}=\alpha_{1}^{2} \frac{\pi^{2}-4}{\pi N} .
$$

Again, to the leading order the bias error is independent of $N$ and can be reduced by increasing $M$. The discretization error dominates, since in this short wavelength limit, there are only two grid cells resolving the wave. Hopefully the energy content of these short wavelength volume fraction variations will be small. Otherwise, grid resolution must increase to accommodate the short wavelength variation. Clearly the particular choices of how many total Lagrangian particles $(N M)$ and how to optimally distribute them between the number of grid cells $(N)$ and the average number of particles per cell $(M)$ depends on the energy content of the different modes of volume fraction variation.

The advantage of the PB methods is that they are not tied to an Eulerian grid. Their averaging width $\delta$ can be chosen independent of the width of the Eulerian grid cell. The error analysis of section 3.2 can be repeated for the top-hat PB method with the $j$-mode volume fraction variation given in (45). The resulting expression for the deterministic error can now be expressed as

$$
\overline{\bar{e}}_{T H}=\frac{1}{M}\left(\frac{N_{p}-1}{N_{p}}\right)+\frac{N_{p}}{M} \frac{\alpha_{1}^{2}}{2 \pi j}\left(1-\frac{2}{N_{p}}\right) \sin \left(\frac{\pi j}{N_{p}}\right)-\frac{N_{p}}{M} \frac{\alpha_{1}^{2}}{2 \pi^{2} j^{2}} \sin ^{2}\left(\frac{\pi j}{N_{p}}\right)+\frac{\alpha_{1}^{2}}{2}\left[1-\frac{N_{p}}{\pi j} \sin \left(\frac{\pi j}{N_{p}}\right)\right]^{2} .
$$

where the sum of the first three terms on the right define the bias error, while the last term is the discretization error. The bias and discretization errors in the long wavelength limit (i.e., small $j$ ) can be obtained as

$$
\overline{\bar{e}}_{B, T H, N_{p} \rightarrow \infty}=\frac{1}{M}\left(1+\frac{1}{2} \alpha_{1}^{2}\right)-\frac{1}{M N_{p}}\left(1+\frac{3}{2} \alpha_{1}^{2}\right) \quad \text { and } \quad \overline{\bar{e}}_{D, T H, N_{p} \rightarrow \infty}=\frac{1}{72} \frac{\alpha_{1}^{2} \pi^{4} j^{4}}{N_{p}^{4}} .
$$


These errors are similar to those presented in (47) for the zeroth order GB method. The biggest difference is in the short wavelength limit of $j=N / 2$ (short wave based on the Eulerian grid). Since the top-hat width is independent of the Eulerian grid, with a large number of Lagrangian particles, $N_{p}$ can be chosen to be much larger than $N$. Thus, provided $N_{p} \gg N$, even in the short wavelength limit equation (50) applies.

The optimal choice of top-hat width (or equivalently optimal $N_{p}$ ) can be evaluated by balancing the bias and discretization errors. If the bias error is taken to be given by the first equation of (50), the optimal value of $N_{p}$ than minimizes the deterministic error (sum of bias and discretization errors) can be obtained as

$$
N_{p, o p t}=\left[\frac{\pi^{4} \alpha_{1}^{2} j^{4}\left(M N_{p}\right)}{18\left(1+\frac{1}{2} \alpha_{1}^{2}\right)}\right]^{1 / 5} .
$$

The optimal value of top-hat width varies only very weakly with the total number of Lagrangian particles $\left(M N_{p}\right)$. The direct dependence on mode number is stronger and goes as $j^{4 / 5}$. However, $\alpha_{1}^{2}$ is a measure of energy in that sinusoidal mode of volume fraction variation and it will depend on the mode number $j$. If the energy spectrum of volume fraction variation decays as some power of $j, N_{p, o p t}$ is expected to be less strongly dependent on $j$. The above arguments apply equally well for all GB methods. The precise optimal choice of top-hat or Gaussian width will depend on the volume fraction variation to be approximated.

\subsection{Sub-grid resolution}

Here consider the scenario where the particle volume fraction variation is on a scale finer than the grid. In other words, volume fraction variation is given by (45), but with $j>N / 2$. In the GB methods there are fewer than two grid cells resolving the sinusoidal volume fraction variation, and thus Nyquist criterion is violated. For simplicity of analysis, attention is restricted to integer number of modes within a grid cell (i.e., $j / N$ to be an integer). As per the analysis of the previous section, the bias error can be decreased by increasing the average number of particles per cell. However, the discretization error will dominate. Since the GB methods are incapable of resolving sub-grid variation, the entire sinusoidal variation will contribute to discretization error, which can be evaluated as

$$
\overline{\bar{e}}=\overline{\bar{e}}_{D}=\alpha_{1}^{2} \frac{\pi}{2} .
$$

To obtain a quantitative estimate of the bias and stochastic errors under sub-grid resolution, an ensemble of numerical tests is performed. Since bias and stochastic errors are dominantly dependent on $M$, in these tests $N=2$, and the error is evaluated for the zeroth, first, and second order GB methods. The deterministic and stochastic error are plotted as a function of $M$ and $1 / \sqrt{M}$ in Fig. 15a and Fig. 15b for several different values of $j$. It is clear for the GB methods under sub-grid resolution that the bias error is as large as discretization error and dominates the stochastic error. 


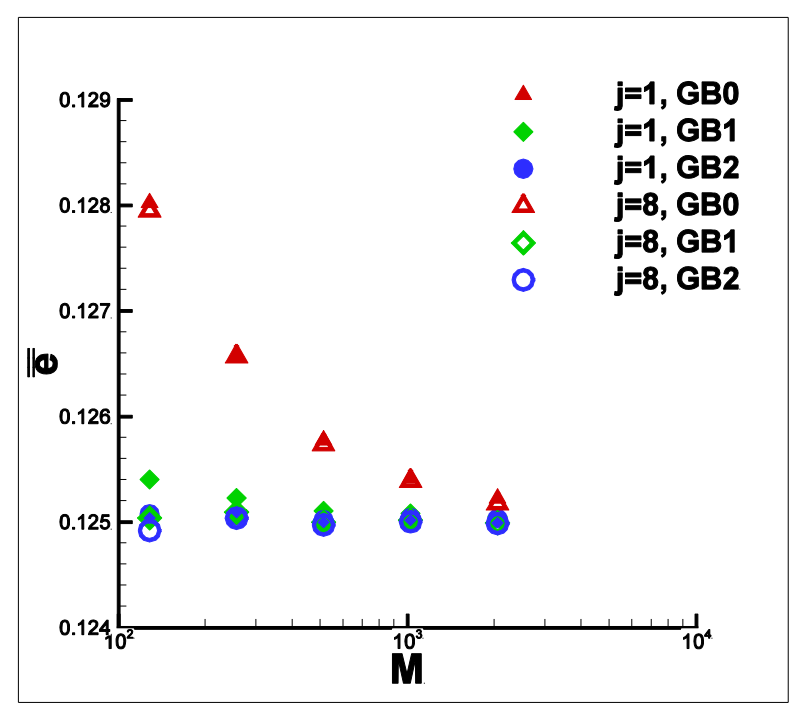

(a)

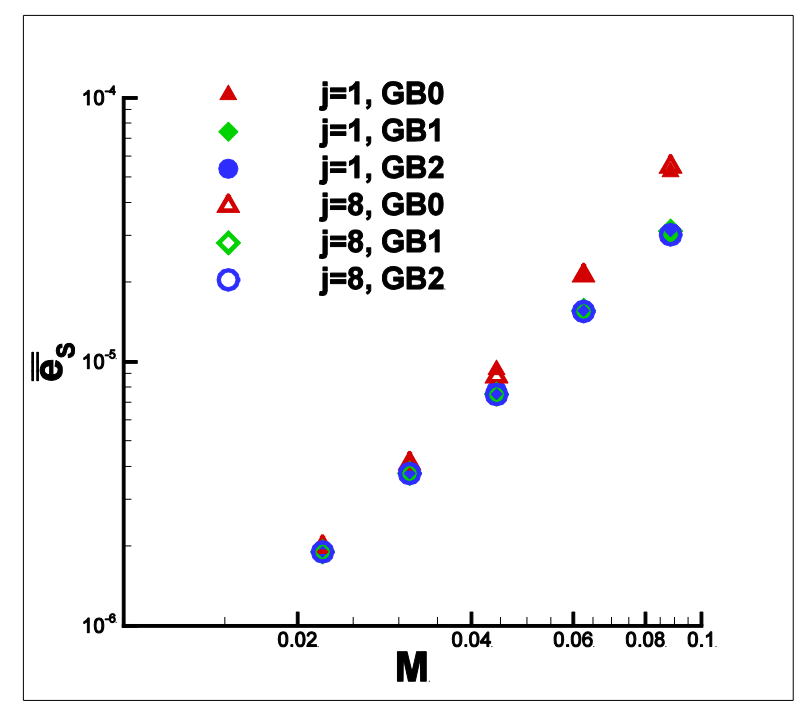

(b)

Fig. 15. (a) Deterministic and (b) stochastic error in the zeroth (GB0), first (GB1) and second (GB2) order GB methods for subgrid resolution with $j=1$ and $j=8, \alpha_{1}=0.5$.

Since PB methods are not tied to the Eulerian grid, sub-grid resolution (resolution of volume fraction variation on scales smaller than the Eulerian grid) is not an issue. For example, for the top-hat PB method the analytical result of (49) applies even for $j>N / 2$. For the top-hat and Gaussian PB methods, the deterministic and stochastic errors are computed numerically for varying values of $j$. When numerically calculating the volume fraction the optimal $\delta$ is used for both the top-hat and Gaussian PB methods. Unlike the GB methods, by increasing the average number of particles per wave $\left(N_{p} M / j\right)$, better approximations to the exact particle volume fraction variation can be obtained for the PB methods, since discretization, bias and stochastic errors are reduced.

In Fig. 16a the discretization error is plotted as a function of the number of waves $j$. The bias error as a function of $1 / N_{p} M$ is shown in Fig. 16b. The PB Gauss method shows slightly higher discretization error, but slightly lower bias error. When the volume fraction estimation for a single realization is compared, the Gauss method produces smoother volume fraction across the domain for a range of $N_{p} M$ and $j$ values. For the top-hat and Gauss PB methods, the stochastic error is plotted as a function of $1 / \sqrt{N_{p} M}$ in Fig. 16c for two different values of $j$. As expected, all methods show an $O\left(1 / \sqrt{N_{p} M}\right)$ decay of the stochastic error with increasing $M$. The PB methods show similar stochastic error. 


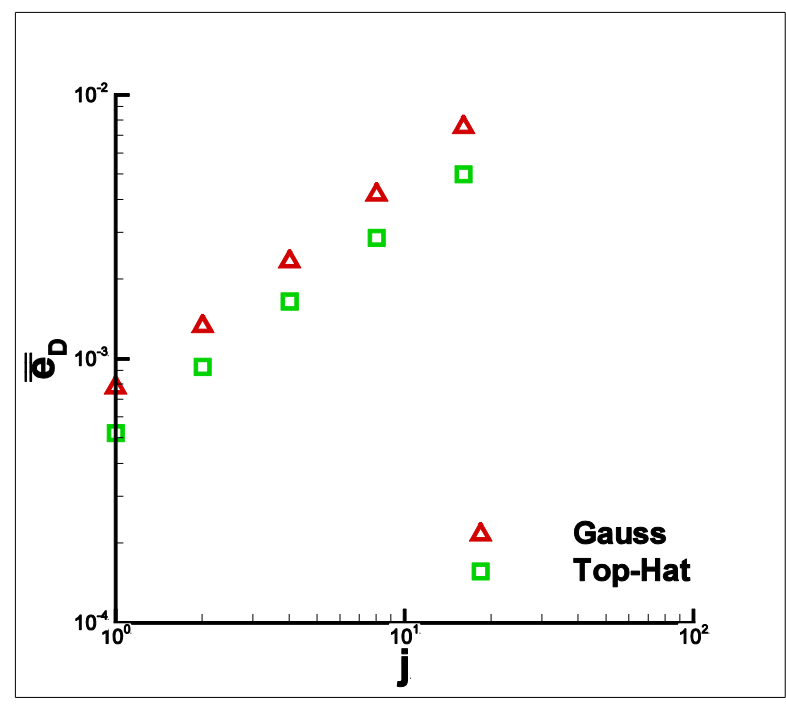

(a)

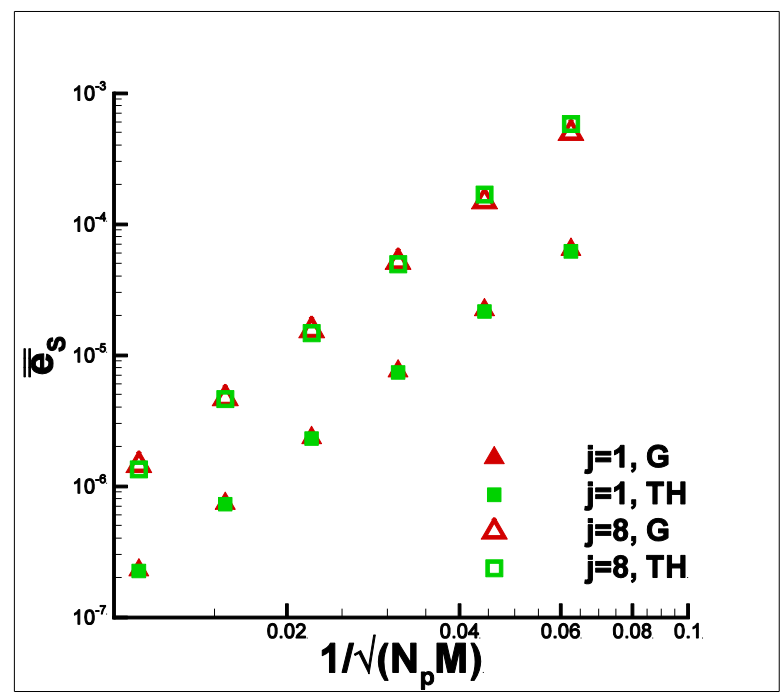

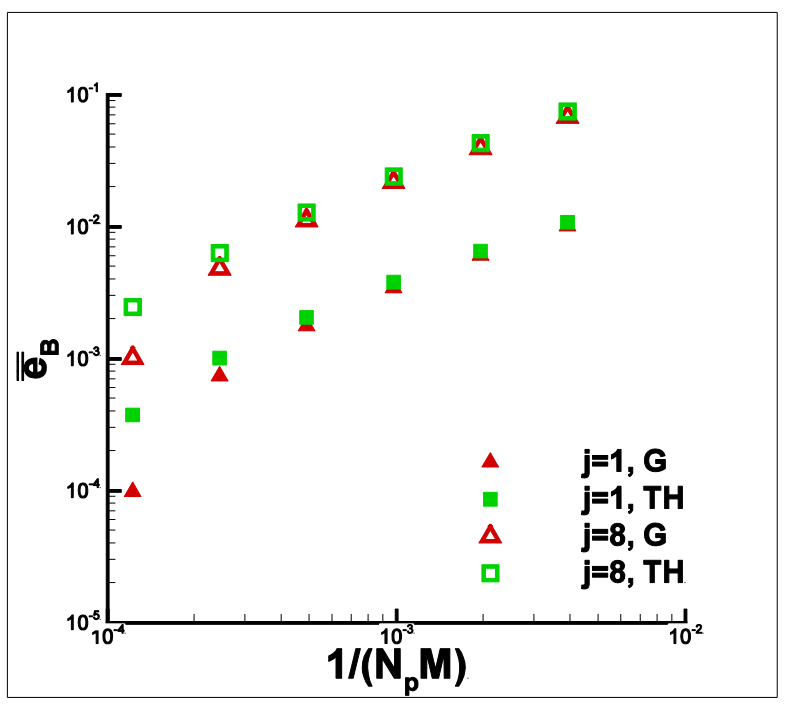

(b)

(c)

Fig. 16. A) Discretization, b) bias and c) stochastic errors for sub-grid implementation of PB methods, $\alpha_{1}=0.5$.

\section{Conclusion}

The deterministic and stochastic errors associated with calculating the particle volume fraction is investigated in this paper through von Neumann error analysis. The multiphase flow community traditionally uses grid-based (GB) methods to calculate particle volume fraction; two particle-based (PB) methods have been developed and studied. GB and PB methods are significantly affected by the width of the averaging region allowed. For GB methods, the width of averaging is determined by the Eulerian grid resolution, $N$. For very small values of $N$, the GB methods are unable to capture sinusoidal volume fraction variation. As $N$ increases, the GB methods yield a much better estimation of the volume fraction. However, as $N$ is further increased beyond an optimal value, the oscillation in the volume fraction estimation, and hence the value of the average mean square error, increases as well. In other 
words, for a fixed total number of particles, an increase of grid cells will be accompanied by a reduction in the number of particles per cell, which will increase both the bias and stochastic errors. This is consistent with the recommendation of Garg et al. [18] that as grid resolution is increased, the number of particles per cell must be maintained.

For PB methods, two distinct methods of calculating local particle volume fraction were considered: the top-hat method and the Gaussian method, both of which are independent of the Eulerian grid. The averaging width of the top-hat and Gauss methods are fixed by the parameter $\delta$. In the present work the optimal value of $\delta$ was found for a given $N_{p} M$ and $\alpha_{1}$. For $\delta$ larger than the optimal value, the averaging width and the number of particles included in the averaging width increases, resulting in a lower bias error, but the sinusoidal volume fraction is poorly approximated and the resulting larger discretization error dominates. On the other hand, for $\delta$ smaller than the optimal value, bias error dominates the discretization error.

For both GB and PB methods, when the average number of particles per cell (or total particles, for Gaussian PB) is increased, the estimation of particle volume fraction is smoother and the deterministic and stochastic errors decrease. In most cases, practitioners can expect the error to continue to decrease as the total number of particles in increased in a given simulation. For sinusoidal oscillations, if the averaging width is too large, there is a point beyond which an increase in the number of particles will not cause a decrease in the average mean squared error, as shown in Fig. 14. The leveling off of the total deterministic error as shown in Fig. 14 is an important point, as some practitioners may increase the number of grid cells or the number of particles per grid cell independently without understanding the effect on the total deterministic error. The Gaussian PB method is particularly attractive for use in multiphase flow computations because the average mean square error decreases as the number of particles increases, regardless of the underlying Eulerian grid.

Additionally, the Gaussian PB method may be used to calculate the particle volume fraction at any location within the flow. Here, the volume fraction has been calculated at the particle locations in order to compare the error at the particle locations. However, with simple modification of equation (21), the particle volume fraction may be calculated at the Eulerian grid cell centers, interfaces, or at any other location of interest. This is particularly powerful for the practitioner in that the particle volume fraction can now be calculated at any location within an Eulerian-Lagrangian flow without the need for the interpolation or projection operations required with traditional GB methods. Because the fluid and particle volume fractions must sum to unity, this also allows to the fluid volume fraction to be calculated with accuracy at any location in the Eulerian-Lagrangian flow. In Eulerian-Lagrangian flow, the fluid volume fraction is typically solved at the Eulerian grid cell center (or interface) and is used to calculate the fluid flux terms. The particle volume fraction is additionally solved at each particle location to calculate to force and heat transfer on each particle. By using the Gaussian PB method at both the Eulerian grid cell centers (or interfaces) and the Lagrangian particle locations, practitioners have a consistent calculation method for volume fraction in both the Eulerian and Lagrangian reference frames.

The ability of the GB and PB methods to estimate sub-grid resolution was briefly considered by introducing multiple waves into the domain. As the number of waves increased, the GB methods were unable to accurately estimate the volume fraction for any value of $N M$ or $\alpha_{1}$. Since PB methods, and their optimal width, are not tied to an Eulerian grid cell, they allow the resolution of any number of waves within the domain, provided there are sufficient averaging widths per wavelength. As the 
sinusoidal component of volume fraction variation decreases, an increased number of particles is required in the domain to define an optimal $N_{p}$, but this is due to deterministic error being dominated by the bias component.

For implementation of the PB methods in a multiphase flow simulation, there are still several challenges that must be carefully considered. First, for the von Neumann analysis presented, cyclic boundary conditions are used. If problems such as a planar curtain of particles subjected to a normal shockinduced flow are considered, the calculation of particle volume fraction at the edges of the particle curtain must be properly implemented for the PB methods. For the case of a dense, planar particle curtain, the sinusoid considered here does not exist, and the optimal averaging width must be determined. In preliminary analysis, the optimal averaging widths presented in Fig. 3 provide a reasonable starting point for analysis of non-sinusoidal conditions. Furthermore, for turbulent flow with a range of scales, the optimal averaging width may be fixed such that larger, more energetic eddy scales are optimally-resolved and smaller, less energetic eddy scales are less well resolved.

Additionally, the top-hat and Gaussian PB methods have similar deterministic and stochastic errors when using the optimal averaging width. However, the Gaussian PB method is the preferred method, as the volume fraction estimation in any individual realization produces a much smoother particle volume fraction variation. The smooth volume fraction variation from particle to particle is required such that the particle forces and heat calculated are smooth, allowing smooth forces and heat to be fed back to the carrier flow.

Though implementation challenges exist, practitioners can expect a reduction in numerical error when using the Gaussian PB method for the calculation of particle volume fraction, or other field representations of Lagrangian quantities.

\section{Acknowledgements}

The authors gratefully acknowledge support from the U.S. Department of Defense Science, Mathematics \& Research for Transformation (SMART) Scholarship and U.S. Department of Energy, National Nuclear Security Administration, Advanced Simulation and Computing Program, as a Cooperative Agreement to the University of Florida under the Predictive Science Academic Alliance Program, under Contract No. DE-NA0002378.

\section{References}

1. K. D. Squires, J.K. Eaton, Particle response and turbulence modification in isotropic turbulence, Phys. Fluids 2 (1990), 1191.

2. S. Elghobashi, G.C. Truesdell, On the two-way interaction between homogeneous turbulence and dispersed solid particles. I: Turbulence modification, Phys. Fluids 5 (1993), 1790.

3. S. Balachandar, J.K. Eaton, Turbulent dispersed multiphase flow, Ann. Rev. of Fluid Mech. 42 (2010), 111.

4. M.J. Andrews, P.J. O'Rourke, The multiphase particle-in-cell (MP-PIC) method for dense particulate flows, Int. J. of Multiphase Flow 22 (2) (1996), 379-402. 
5. D.M. Snider, P.J. O'Rourke, M.J. Andrews, Sediment flow in inclined vessels calculated using a multiphase particle-in-cell model for dense particle flows, Int. J. Multiphase Flow 24 (8) (1998), 1359-1382.

6. D.M. Snider, An incompressible three-dimensional multiphase particle-in-cell model for dense particle flows, J. Comp. Phys. 170 (2) (2001), 523-549.

7. N.A. Patankar, D.D. Joseph, Modeling and numerical simulation of particulate flows by the Eulerian-Lagrangian approach, Int. J. Multiphase Flow 27 (10) (2001), 1659-1684.

8. S. V. Apte, K. Mahesh, T. Lundgren, Accounting for finite-size effects in simulations of disperse particle-laden flows, Int. J. Multiphase Flow 34 (3) (2008), 260-271.

9. S. Elghobashi, Particle-laden turbulent flows: direct simulation and closure models, Appl. Sci. Res. 48 (3-4) (1991), 301-314.

10. S. Elghobashi, On predicting particle-laden turbulent flows, Appl. Sci. Res. 52 (4) (1994), 309-329.

11. S. Balachandar, M.R. Maxey, Methods for evaluating fluid velocities in spectral simulations of turbulence, J. Comp. Phys. 83 (1989), 96-125.

12. P.K. Yeung, S.B. Pope, An algorithm for tracking fluid particles in numerical simulations of homogeneous turbulence, J. Comp. Phys. 79 (1988), 373-416.

13. M.W. Evans, F.H. Harlow, E. Bromberg, The Particle-in-Cell method for hydrodynamic calculations, Los Alamos National Lab, NM, No. LA-2139, (1957).

14. F.H. Harlow, PIC and its progeny, Comp. Phys. Comm. 48 (1988), 1-10.

15. S. Sundaram, L.R. Collins, Considerations in simulating a turbulent suspension of finite-volume particles, J. Comp. Phys. 124 (1996), 337-350.

16. M. Boivin, O. Simonin, K.D. Squires, Direct numerical simulation of turbulence modulation by particles in isotropic turbulence, J. Fluid Mech. 375 (1998), 235-263.

17. C. Narayanan, D. Lakehal, G. Yadigaroglu, Linear stability analysis of particle-laden mixing layers using Lagrangian particle tracking, Powder Tech. 125 (2002), 122-130.

18. R. Garg, C. Narayanan, D. Lakehal, S. Subramaniam, Accurate numerical estimation of interphase momentum transfer in Lagrangian-Eulerian simulations of dispersed two-phase flows, Int. J. Multiphase Flow 33 (12) (2007), 1337-1364.

19. M. Garcia-Villalba, A.G. Kidanemariam, M.Uhlmann, DNS of vertical plane channel flow with finite-size particles: Voronoi analysis, acceleration statistics and particle-conditioned averaging, Int. J. Multiphase Flow 46 (2012), 54-74.

20. R. Garg, C. Narayanan, S. Subramaniam, A numerically convergent Lagrangian-Eulerian simulation method for dispersed two-phase flows, Int. J. Multiphase Flow 35 (4) (2009), 376388.

21. D. Stoyan, W.S. Kendall, J. Meinke, Stochastic geometry and its applications, Akadenie-Verlag, Berlin (1995).

22. S. Subramaniam, Statistical representation of a spray as a point process, Phys. of Fluids 12 (10) (2000), 2413-2431.

23. S. Subramaniam, Statistical modeling of sprays using the droplet distribution function, Phys. of Fluids 13 (3) (2001), 624-642.

24. S. Subramaniam, Lagrangian-Eulerian methods for multiphase flows, Prog. in Energy and Combust. Sci. 39 (2) (2013), 215-245. 
25. E. Balkovsky, G. Falkovich, A. Fouxon, Intermittent distribution of inertial particles in turbulent flows, Phys. Rev. Lett. 86 (13) (2001), 2790.

26. F. Toschi, E. Bodenschatz, Lagrangian properties of particles in turbulence, Annu. Rev. Fluid Mech. 41 (2009), 375-404.

27. M. Roma, C.S. Peskin, M.J. Berger, An adaptive version of the immersed boundary method, J. Comp. Phys. 153 (2) (1999), 509-534.

28. M. UhImann, An immersed boundary method with direct forcing for the simulation of particulate flows, J. Comp. Phys. 209 (2) (2005), 448-476.

29. R. Mittal, G. laccarino, Immersed boundary methods, Annu. Rev. Fluid Mech. 37 (2005), 239261.

30. E. Onate, S. Idelsohn, A mesh-free finite point method for advective-diffusive transport and fluid flow problems, Comp. Mech. 21 (4-5) (1998), 283-292.

31. J. Xu, S.B. Pope, Assessment of numerical accuracy of pdf/Monte Carlo methods for turbulent reacting flows, J. Comp. Phys. 152 (1999), 192-230.

32. S.B. Pope, Particle method for turbulent flows: integration of stochastic model equations, J. Comp. Phys. 117 (2) (1995), 332-349

33. G. Casella and R. L. Berger, Statistical Inference (Duxbury, Pacific Grove, CA, 2002). 